\title{
The NINJA-2 catalog of hybrid post-Newtonian/numerical-relativity waveforms for non-precessing black-hole binaries
}

\author{
P. Ajith, ${ }^{1}$ Michael Boyle, ${ }^{2}$ Duncan A. Brown, ${ }^{3}$ Bernd \\ Brügmann, ${ }^{4}$ Luisa T. Buchman, ${ }^{5}$ Laura Cadonati, ${ }^{6}$ \\ Manuela Campanelli, ${ }^{7}$ Tony Chu, ${ }^{5,8}$ Zachariah B. Etienne, ${ }^{9}$ \\ Stephen Fairhurst, ${ }^{10}$ Mark Hannam, ${ }^{10}$ James Healy, ${ }^{11}$ Ian \\ Hinder, ${ }^{12}$ Sascha Husa, ${ }^{13}$ Lawrence E. Kidder, ${ }^{2}$ Badri \\ Krishnan, ${ }^{14}$ Pablo Laguna, ${ }^{11}$ Yuk Tung Liu, ${ }^{9}$ Lionel \\ London, ${ }^{11}$ Carlos O. Lousto, ${ }^{7}$ Geoffrey Lovelace, ${ }^{2}$ Ilana \\ MacDonald, ${ }^{8}$ Pedro Marronetti, ${ }^{15}$ Satya Mohapatra, ${ }^{6}$ \\ Philipp Mösta, ${ }^{12}$ Doreen Müller, ${ }^{4}$ Bruno C. Mundim, ${ }^{7}$ \\ Hiroyuki Nakano, ${ }^{7}$ Frank Ohme, ${ }^{12}$ Vasileios Paschalidis, ${ }^{9}$ \\ Larne Pekowsky, ${ }^{3,11}$ Denis Pollney, ${ }^{13}$ Harald P. Pfeiffer, ${ }^{8}$ \\ Marcelo Ponce, ${ }^{7}$ Michael Pürrer, ${ }^{16}$ George Reifenberger, ${ }^{15}$ \\ Christian Reisswig, ${ }^{5}$ Lucía Santamaría, ${ }^{1}$ Mark A. Scheel, ${ }^{5}$ \\ Stuart L. Shapiro, ${ }^{9}$ Deirdre Shoemaker, ${ }^{11}$ Carlos F. \\ Sopuerta, ${ }^{17}$ Ulrich Sperhake, ${ }^{17,5,18,19}$ Béla Szilágyi, ${ }^{5}$ \\ Nicholas W. Taylor, ${ }^{5}$ Wolfgang Tichy, ${ }^{15}$ Petr Tsatsin, ${ }^{15}$ \\ and Yosef Zlochower ${ }^{7}$ \\ ${ }^{1}$ LIGO - California Institute of Technology, Pasadena, California 91125, USA \\ ${ }^{2}$ Center for Radiophysics and Space Research, Cornell University, Ithaca, New \\ York, 14853, USA \\ ${ }^{3}$ Department of Physics, Syracuse University, Syracuse, New York, 13254, USA \\ ${ }^{4}$ Theoretisch Physikalisches Institut, Friedrich Schiller Universität, 07743 Jena, \\ Germany \\ ${ }^{5}$ Theoretical Astrophysics 130-33, California Institute of Technology, Pasadena, \\ California 91125, USA \\ ${ }^{6}$ Department of Physics, University of Massachusetts, Amherst, Massachusetts \\ 01003, USA \\ ${ }^{7}$ Center for Computational Relativity and Gravitation and School of \\ Mathematical Sciences, Rochester Institute of Technology, 85 Lomb Memorial \\ Drive, Rochester, New York 14623, USA \\ ${ }^{8}$ Canadian Institute for Theoretical Astrophysics, University of Toronto, \\ 60 St. George Street, Toronto, Ontario M5S 3H8, Canada \\ ${ }^{9}$ Department of Physics, University of Illinois at Urbana-Champaign, Urbana, \\ Illinois 61801, USA \\ ${ }^{10}$ School of Physics and Astronomy, Cardiff University, The Parade, Cardiff, UK \\ ${ }^{11}$ Center for Relativistic Astrophysics and School of Physics, Georgia Institute \\ of Technology, Atlanta, Georgia 30332, USA \\ ${ }^{12}$ Max-Planck-Institut für Gravitationsphysik, Albert-Einstein-Institut, Am \\ Mühlenberg 1, D-14476 Golm, Germany \\ ${ }^{13}$ Departament de Física, Universitat de les Illes Balears, Crta. Valldemossa km \\ 7.5, E-07122 Palma, Spain \\ ${ }^{14}$ Max-Planck-Institut für Gravitationsphysik, Albert-Einstein-Institut, \\ Callinstraße 38, D-30167 Hannover, Germany \\ ${ }^{15}$ Department of Physics, Florida Atlantic University, Boca Raton, Florida \\ 33431
}




\author{
${ }^{16}$ Gravitational Physics, Faculty of Physics, University of Vienna, \\ Boltzmanngasse 5, A-1090 Vienna, Austria \\ ${ }^{17}$ Institut de Ciencies de l'Espai (CSIC-IEEC), Campus UAB, Bellaterra, 08193 \\ Barcelona, Spain \\ ${ }^{18}$ Department of Physics and Astronomy, The University of Mississippi, \\ University, Mississippi 38677, USA \\ ${ }^{19}$ CENTRA, Departamento de Física, Instituto Superior Técnico, Av. Rovisco \\ Pais 1, 1049-001 Lisboa, Portugal
}

\begin{abstract}
The Numerical INJection Analysis (NINJA) project is a collaborative effort between members of the numerical relativity and gravitational wave data analysis communities. The purpose of NINJA is to study the sensitivity of existing gravitational-wave search and parameter-estimation algorithms using numerically generated waveforms, and to foster closer collaboration between the numerical relativity and data analysis communities. The first NINJA project used only a small number of injections of short numerical-relativity waveforms, which limited its ability to draw quantitative conclusions. The goal of the NINJA2 project is to overcome these limitations with long post-Newtonian-numerical relativity hybrid waveforms, large numbers of injections, and the use of real detector data. We report on the submission requirements for the NINJA-2 project and the construction of the waveform catalog. Eight numerical relativity groups have contributed 63 hybrid waveforms consisting of a numerical portion modelling the late inspiral, merger, and ringdown stitched to a post-Newtonian portion modelling the early inspiral. We summarize the techniques used by each group in constructing their submissions. We also report on the procedures used to validate these submissions, including examination in the time and frequency domains and comparisons of waveforms from different groups against each other. These procedures have so far considered only the $(\ell, m)=(2,2)$ mode. Based on these studies we judge that the hybrid waveforms are suitable for NINJA-2 studies. We note some of the plans for these investigations.
\end{abstract}

\title{
1. Introduction
}

A new generation of laser interferometric gravitational-wave detectors (Advanced LIGO [1-3], Advanced Virgo [4,5], and LCGT [6]) is presently under construction. These second-generation detectors will have an order of magnitude increase in sensitivity over first generation instruments and will be sensitive to a broader range of gravitational-wave frequencies. One of the most widely anticipated sources for this global network of observatories is the inspiral, merger and ringdown of a binary containing two black holes [7]. Detection of such a binary black hole coalescence will allow astronomers and astrophysicists to directly observe the physics of black-hole spacetimes and to explore the strong-field conditions of Einstein's theory of general relativity [8].

The ability of gravitational-wave astronomers to use the new generation of observatories to detect and study binary black hole coalescence depends on the quality of search and source-parameter measurement algorithms. These algorithms rely on the physical accuracy of the underlying theoretical waveform models. Developing and testing the algorithms required to achieve the goals of gravitational-wave astronomy demands close interaction between the source-modeling and data-analysis communities. The Numerical INJection Analysis (NINJA) project was created in 2008 to bring these communities together and to use the recent advances in numerical relativity (NR) [9] to test analysis pipelines by adding physically realistic signals 
to detector noise in software. We describe such additions of signals into noise as "injections."

The first NINJA project (NINJA-1) [10] considered a total of 23 numerical waveforms, which were injected into Gaussian noise colored with the frequency sensitivity of first-generation detectors. These data were analyzed by nine dataanalysis groups using both search and parameter-estimation algorithms. However, there were two major limitations to the NINJA-1 analysis: First, to encourage broad participation, no length or accuracy requirements were placed on the numerical waveforms. Consequently, many of these waveforms were too short to inject over an astrophysically interesting mass range without introducing artifacts into the data. The lowest mass binary considered in NINJA-1 had a total mass of $35 \mathrm{M}_{\odot}$, whereas the mass of black holes could extend below $5 \mathrm{M}_{\odot}[11,12]$. In NINJA-1, the waveforms were only inspected for obvious, pathological errors and no cross-checks were performed between the submitted waveforms; and, therefore it was difficult to assess the physical fidelity of the results. Second, the NINJA-1 data set contained stationary noise with the simulated signals already injected into the data. The data set contained only 126 simulated signals, which precluded detailed statistical studies of the effectiveness of search and parameter estimation algorithms. Finally, since the data set lacked the non-Gaussian noise transients present in real detector data, it was not possible to fully explore the response of the algorithms in a real search scenario. Despite these limitations, NINJA-1 successfully removed a number of barriers to collaboration between the source-modelling and data-analysis communities and demonstrated where further work is needed. The goal of the second NINJA project (NINJA-2) is to address the deficiencies of NINJA-1 and to perform a systematic test of the efficacy of dataanalysis pipelines in real-world situations in preparation for Advanced LIGO and Virgo.

This paper reports on the improvements we have made to the NINJA analysis to address the first of the limitations described above - the accuracy of the numerical waveforms. We present the NINJA-2 waveform catalog and describe the results of the procedures we have used to validate these data. NINJA-2 places requirements on the accuracy and length of the contributed waveforms and we have performed systematic cross-checks of the submitted waveforms. Each binary black hole simulation in the NINJA-2 catalog must include at least five orbits of usable data before merger, i.e., neglecting the initial burst of junk radiation. The NR waveform amplitude should be accurate to within $5 \%$, and the phase (as a function of gravitational-wave frequency) should have an accumulated uncertainty over the entire inspiral, merger and ringdown (of the numerical simulation), of no more than $0.5 \mathrm{rad}$. We also require that numerical simulations are "hybridized" to post-Newtonian $(\mathrm{pN})$ waveforms so that the resulting waveforms contain enough cycles to allow injections at $M \geq 10 \mathrm{M}_{\odot}$ in early Advanced LIGO data. The continued advances in numerical simulations have also allowed us to study a somewhat larger region of the signal parameter space; however we have restricted our attention to non-precessing binaries for reasons discussed in Sec. 2 below.

A subsequent paper will describe the results of using the NINJA-2 waveforms to study search and parameter estimation algorithms in real detector data. Since data from the second-generation detectors is not yet available, the NINJA-2 analysis will use data from the first-generation detectors re-colored so that it has the frequency response expected in the first observing runs of the Advanced LIGO (aLIGO) detectors - referred to as "early aLIGO" [13]. Similar noise curves will be used to simulate the Advanced Virgo detector. NINJA-2 analyses will use these noise models to ensure 


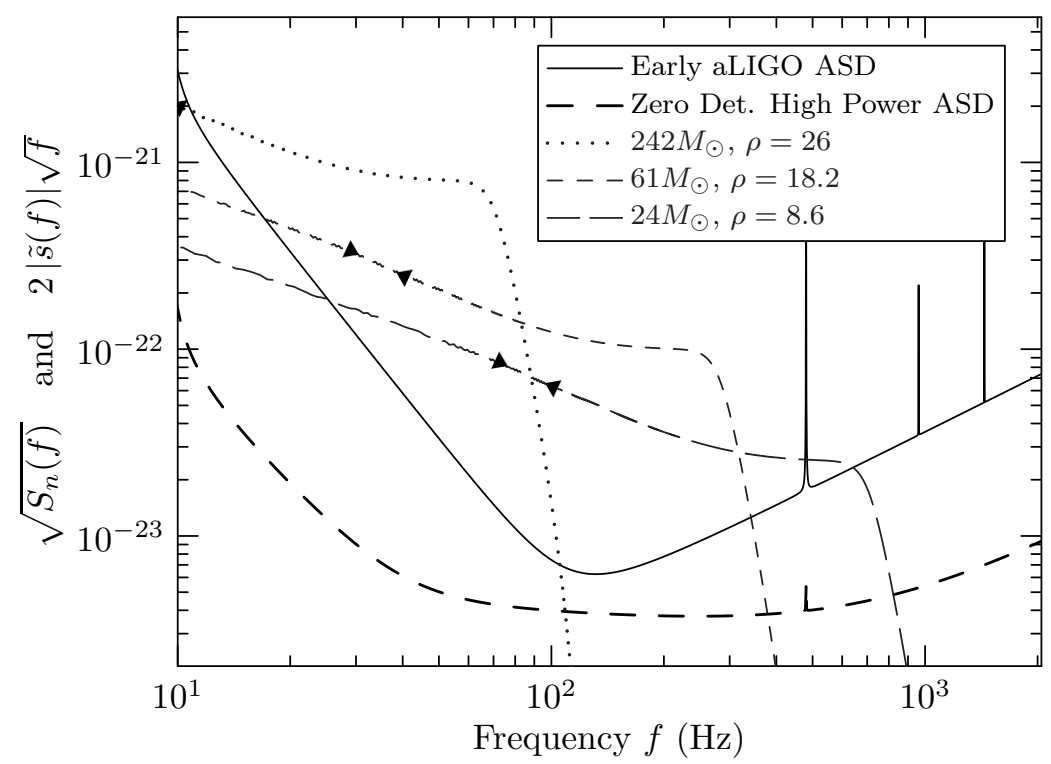

Figure 1. The hybrid $q=m_{1} / m_{2}=2$, non-spinning MayaKranc waveform scaled to various total masses shown against the early and zero-detuned-highpower aLIGO noise curves, shown as amplitude spectral densities, the square root of the power spectral densities. The triangles represent the starting and ending frequencies of the post-Newtonian hybridization region, given in table 1 . The total mass of the binary is scaled so that the hybridization region ends at $100 \mathrm{~Hz}$, $40 \mathrm{~Hz}$, and $10 \mathrm{~Hz}$. The amplitude of the signal is scaled so that it represents an optimally oriented binary at a distance of $1 \mathrm{Gpc}$ from the detector. The early aLIGO sensitivity is used to compute the signal-to-noise ratio $\rho$.

that existing algorithms are optimal when second-generation detectors come online in $\sim 2015$. The results in this paper use the early aLIGO sensitivity curve (cf. Fig. 1) to study the accuracy of the submitted waveforms. The ultimate sensitivity of Advanced LIGO is expected to be significantly better than this curve. To allow the waveforms to be used in studies using more sensitive noise curves, we have also performed accuracy studies using the aLIGO zero-detuned, high-power [14] sensitivity curve. Fig. 1 shows the two aLIGO sensitivity curves, characterized by their Amplitude Spectral Densities (ASD) overlaid with one of the contributed NINJA-2 waveforms. This figure demonstrates that hybridization is necessary to allow scaling of the numerical waveforms to astrophysically interesting masses, and a portion of the present paper studies the hybridization methods used to construct the NINJA-2 waveforms.

The remainder of this paper is organized as follows: Section 2 describes in more detail the accuracy requirements that we have placed on NINJA-2 simulations and presents an overview of the waveform catalog showing the regions of the binary black hole parameter space covered. Section 3 gives an overview of the numerical methods used to construct the numerical relativity waveforms and Sec. 4 describes the methods that we have used to hybridize the numerical simulations to $\mathrm{pN}$ waveforms. The $\mathrm{pN}$ waveforms themselves are summarized in the Appendix. Section 5 describes the methods and results of the comparisons we have performed between the waveforms. Based on these comparisons, we judge the hybrid waveforms suitable for the NINJA2 project. Section 6 summarizes our findings and suggests directions for future 
improvements of the catalog, in particular the study of higher order modes in the waveforms.

\section{Overview of the Waveform Catalog}

Binary black holes formed from the evolution of massive stars are expected to have circularized before their gravitational-wave frequency reaches the sensitive band of ground-based detectors, and so we only consider circular (non-eccentric) binaries. In the NINJA-2 project we further restrict our attention to binaries that do not undergo precession, i.e., where the spins of the black holes either vanish or are parallel (or anti-parallel) to the binary's orbital angular momentum. We do this for two reasons: (i) in trying to understand the complex phenomenology of the binary parameter space, we prefer to tackle first a simpler subset, which nonetheless captures the main features of binary inspiral and merger; (ii) the precessing-binary parameter space has been sampled by only a handful of numerical simulations. The numerical-relativity community is currently exploring the space of precessing binaries, for example through the numerical relativity-analytical relativity (NRAR) project [15]. Such waveforms will be used in future NINJA projects that explore precession.

The parameters of the black-hole binaries we consider in NINJA-2 are the mass of each black hole, $m_{1}$ and $m_{2}$, or equivalently the total mass $M=m_{1}+m_{2}$ and mass ratio $q=m_{1} / m_{2}$, and the dimensionless spin-magnitude of each black hole, $\chi_{1} \equiv S_{1} / m_{1}^{2}$ and $\chi_{2}=S_{2} / m_{2}^{2}$. The total mass sets the overall scale of the system, and can be factored out to leave a three-dimensional parameter space, $\left\{q, \chi_{1}, \chi_{2}\right\}$. Figure 2 shows the coverage of parameter space (details in Sec. 3). The sampling is coarse; while the waveforms will provide invaluable information within the NINJA-2 project, we expect that ultimately a more uniform coverage of parameter-space by a much larger number of configurations will be necessary.

The NINJA-2 requirement of five pre-merger orbits is at the low end of estimates of sufficient waveform lengths for the construction of accurate hybrid PNNR waveforms, as discussed in [16-20], but we expect these to be acceptable for the goals of the NINJA-2 project. The $5 \%$ amplitude and $0.5 \mathrm{rad}$ phase accuracy requirements were formulated with typical current waveforms in mind, for example those studied in the Samurai project [21] and studies performed in preparation for the NR-AR collaboration project. These requirements are consistent for waveforms of similar lengths but may not be directly applicable to much longer waveforms. For example, in the 25-orbit SpEC simulations with dimensionless spins $\chi_{i}=0.97$ the highest- and second-highest-resolution data differ by roughly $0.6 \mathrm{rad}$ at merger [22]. Note that because this phase-error accumulates over 20 additional inspiral orbits, this waveform would easily satisfy the NINJA-2 phase requirement if it were truncated to minimally meet the NINJA-2 length requirement (although such a truncation would decrease the accuracy of the hybridized waveform).

We require that the hybridized waveforms in the NINJA-2 catalog are long enough to span the sensitivity bands of the advanced LIGO and Virgo detectors in their early operation. Specifically, when rescaled to $10 \mathrm{M}_{\odot}$ the hybrids must begin with a gravitational wave frequency of $20 \mathrm{~Hz}$ or lower, i.e. a starting $\mathrm{GW}$ frequency of $M \omega \leq 0.006$. This requires extending the NR waveforms to lower frequencies (i.e. more inspiral cycles) by attaching a $\mathrm{pN}$ inspiral waveform onto the early portion of the NR waveform to produce a hybrid $\mathrm{pN}-\mathrm{NR}$ waveform. We require that the hybridization be performed at a gravitational-wave frequency of $M \omega_{22} \leq 0.075$, where 


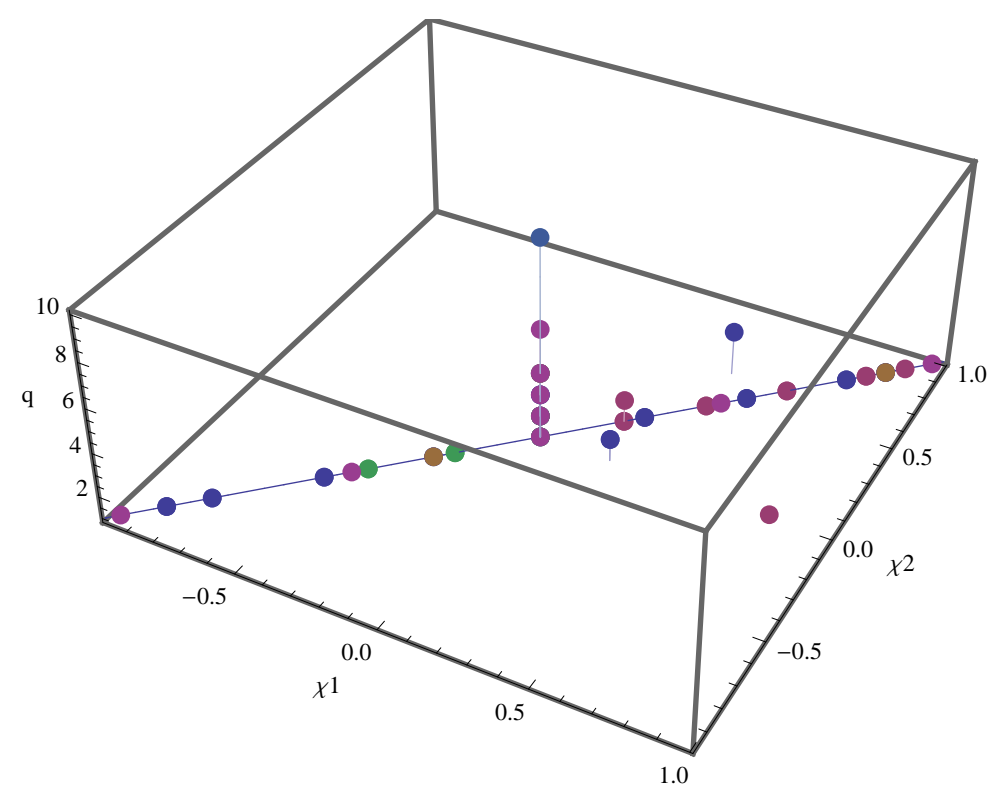

Figure 2. Mass ratio $q$ and dimensionless spins $\chi_{i}$ of the NINJA-2 hybrid waveform submissions.

$M \omega_{22}$ is the frequency of the $(\ell, m)=(2, \pm 2)$ harmonic. In practice, hybridization fits were performed over a frequency range as summarized in Sec. 4, and the average frequency, and frequency of the average time of the fitting interval were always chosen below $M \omega_{22} \leq 0.075$, with two exceptions as seen in Table 1: The nonspinning Llama waveforms at mass ratios $q=1,2$. As seen in Fig. (7) these do however show excellent overlaps with comparison waveforms.

The waveforms were submitted with the complex GW strain function $h_{+}-i h_{\times}$ decomposed into modes using spin-weighted spherical harmonics ${ }^{-2} Y_{\ell m}$ of weight $s=-2$. Although most of the power is in the $(\ell, m)=(2, \pm 2)$ modes, we encouraged (but did not require), the submission of additional subdominant modes. The accuracy studies in this paper focus on the $(\ell, m)=(2,2)$ mode; further work is required to study the accuracy of the contributed subdominant modes. A total of 63 waveforms from 8 groups were contributed to the NINJA-2 catalog. There are 46 distinct numerical waveforms; some of these waveforms have been hybridized with multiple $\mathrm{pN}$ waveforms. The NINJA-2 catalog is summarized in Table 1, and a map of the parameter values is shown in Fig. 2. In the next section, we describe in more detail the numerical methods used to generate these waveforms and present additional plots in Figs. 3 and 4.

\section{Numerical Methods}

\subsection{Summary of contributions}

The NINJA-2 data set contains both hybrid and original numerical relativity waveforms, in a data format that is summarized in Sec. 3.2 below, and described in detail in Ref. [60]. The contributed waveforms cover 29 different black hole 


\begin{tabular}{|c|c|c|c|c|c|c|c|}
\hline$q$ & $\chi_{1}$ & $\chi_{2}$ & Submission & $1000 e$ & $\begin{array}{r}100 M \omega \\
\text { hyb.range }\end{array}$ & $\begin{array}{l}\# \text { NR } \\
\text { cycles }\end{array}$ & $\begin{array}{c}\mathrm{pN} \\
\text { Approx }\end{array}$ \\
\hline 1.0 & -0.95 & -0.95 & SpEC [22-31] & 1.00 & $3.3-4.1$ & 18.42 & $\mathrm{~T} 1$ \\
\hline 1.0 & -0.85 & -0.85 & BAM [32-37] & 2.50 & $4.1-4.7$ & 12.09 & $\mathrm{~T} 1, \mathrm{~T} 4$ \\
\hline 1.0 & -0.75 & -0.75 & BAM [32-37] & 1.60 & $4.1-4.7$ & 13.42 & $\mathrm{~T} 1, \mathrm{~T} 4$ \\
\hline 1.0 & -0.50 & -0.50 & BAM [32-37] & 2.90 & $4.3-4.7$ & 15.12 & $\mathrm{~T} 1, \mathrm{~T} 4$ \\
\hline 1.0 & -0.44 & -0.44 & $\mathrm{SpEC}[23,24,27-30,38]$ & 0.04 & $4.3-5.3$ & 13.47 & $\mathrm{~T} 4$ \\
\hline 1.0 & -0.40 & -0.40 & Llama $[39-41]$ & & $6.1-8.0$ & 6.42 & $\mathrm{~T} 1, \mathrm{~T} 4$ \\
\hline 1.0 & -0.25 & -0.25 & BAM [32-37] & 2.50 & $4.5-5.0$ & 15.15 & $\mathrm{~T} 1, \mathrm{~T} 4$ \\
\hline 1.0 & -0.20 & -0.20 & Llama [39-41] & & $5.7-7.8$ & 8.16 & $\mathrm{~T} 1, \mathrm{~T} 4$ \\
\hline \multirow[t]{4}{*}{1.0} & 0.00 & 0.00 & BAM [32-37] & 1.80 & $4.6-5.1$ & 15.72 & $\mathrm{~T} 1, \mathrm{~T} 4$ \\
\hline & & & GATech [42-48] & 3.00 & $5.5-7.5$ & 9.77 & $\mathrm{~T} 4$ \\
\hline & & & Llama $[40,41]$ & & $5.7-9.4$ & 8.30 & $\mathrm{~F} 2$ \\
\hline & & & SpEC $[23,24,27-30]$ & 0.05 & $3.6-4.5$ & 22.98 & $\mathrm{~T} 4$ \\
\hline 1.0 & 0.20 & 0.20 & GATech [42-48] & 10.00 & $6.0-7.5$ & 10.96 & $\mathrm{~T} 4$ \\
\hline 1.0 & 0.25 & 0.25 & BAM [32-37] & 6.10 & $4.6-5.0$ & 18.00 & $\mathrm{~T} 1, \mathrm{~T} 4$ \\
\hline \multirow[t]{2}{*}{1.0} & 0.40 & 0.40 & GATech [42-48] & 10.00 & $5.9-7.5$ & 12.31 & $\mathrm{~T} 4$ \\
\hline & & & Llama [39-41] & & $7.8-8.6$ & 6.54 & $\mathrm{~T} 1, \mathrm{~T} 4$ \\
\hline 1.0 & 0.44 & 0.44 & $\mathrm{SpEC}[23,24,27-30,38]$ & 0.02 & $4.1-5.0$ & 22.39 & $\mathrm{~T} 4$ \\
\hline 1.0 & 0.50 & 0.50 & BAM $[32-37]$ & 6.10 & $5.2-5.9$ & 15.71 & $\mathrm{~T} 1, \mathrm{~T} 4$ \\
\hline 1.0 & 0.60 & 0.60 & GATech [42-48] & 12.00 & $6.0-7.5$ & 13.63 & $\mathrm{~T} 4$ \\
\hline 1.0 & 0.75 & 0.75 & BAM [32-37] & 6.00 & $6.0-7.0$ & 14.03 & $\mathrm{~T} 1, \mathrm{~T} 4$ \\
\hline 1.0 & 0.80 & 0.00 & GATech [42-48] & 13.00 & $5.5-7.5$ & 12.26 & $\mathrm{~T} 4$ \\
\hline 1.0 & 0.80 & 0.80 & GATech [42-48] & 6.70 & $5.5-7.5$ & 15.05 & $\mathrm{~T} 4$ \\
\hline \multirow[t]{2}{*}{1.0} & 0.85 & 0.85 & BAM [32-37] & 5.00 & $5.9-6.9$ & 15.36 & $\mathrm{~T} 1, \mathrm{~T} 4$ \\
\hline & & & UIUC [49] & 20.00 & $5.9-7.0$ & 15.02 & $\mathrm{~T} 1$ \\
\hline 1.0 & 0.90 & 0.90 & GATech [42-48] & 3.00 & $5.8-7.5$ & 15.05 & $\mathrm{~T} 4$ \\
\hline 1.0 & 0.97 & 0.97 & $\mathrm{SpEC}[22-25,27-31]$ & 0.60 & $3.2-4.3$ & 38.40 & $\mathrm{~T} 4$ \\
\hline \multirow[t]{4}{*}{2.0} & 0.00 & 0.00 & BAM [32-37] & 2.30 & $6.3-7.8$ & 8.31 & $\mathrm{~T} 1, \mathrm{~T} 4$ \\
\hline & & & GATech [42-48] & 2.50 & $5.5-7.5$ & 10.42 & $\mathrm{~T} 4$ \\
\hline & & & Llama $[40,41]$ & & $6.3-9.4$ & 7.47 & $\mathrm{~F} 2$ \\
\hline & & & $\mathrm{SpEC}[23-25,27-31,50]$ & 0.03 & $3.8-4.7$ & 22.34 & $\mathrm{~T} 2$ \\
\hline 2.0 & 0.20 & 0.20 & GATech [42-48] & 10.00 & $5.6-7.5$ & 11.50 & $\mathrm{~T} 4$ \\
\hline 2.0 & 0.25 & 0.00 & BAM $[32,36]$ & 2.00 & $5.0-5.6$ & 15.93 & $\mathrm{~T} 1, \mathrm{~T} 4$ \\
\hline \multirow[t]{2}{*}{3.0} & 0.00 & 0.00 & BAM $[32-37]$ & 1.60 & $6.0-7.1$ & 10.61 & $\mathrm{~T} 1, \mathrm{~T} 4$ \\
\hline & & & SpEC $[23-25,27-31,50]$ & 0.02 & $4.1-5.2$ & 21.80 & $\mathrm{~T} 2$ \\
\hline 3.0 & 0.60 & 0.40 & FAU $[51-54]$ & 1.00 & $5.0-5.6$ & 18.89 & $\mathrm{~T} 4$ \\
\hline \multirow[t]{3}{*}{4.0} & 0.00 & 0.00 & BAM $[32-37]$ & 2.60 & $5.9-6.8$ & 12.38 & $\mathrm{~T} 1, \mathrm{~T} 4$ \\
\hline & & & LEAN $[55,56]$ & 5.00 & $5.1-5.5$ & 17.33 & $\mathrm{~T} 1$ \\
\hline & & & SpEC $[23-25,27-31,50]$ & 0.03 & $4.4-5.5$ & 21.67 & $\mathrm{~T} 2$ \\
\hline 6.0 & 0.00 & 0.00 & SpEC $[23-25,27-31,50]$ & 0.04 & $4.1-4.6$ & 33.77 & $\mathrm{~T} 1$ \\
\hline 10.0 & 0.00 & 0.00 & RIT $[57-59]$ & 0.40 & $7.3-7.4$ & 14.44 & $\mathrm{~T} 4$ \\
\hline
\end{tabular}

Table 1. Summary of the NINJA-2 waveform catalog. Given are mass-ratio $q=m_{1} / m_{2}$, magnitude of the dimensionless spins $\chi_{i}=S_{i} / m_{i}^{2}$, numerical code, orbital eccentricity $e$, frequency range of hybridization in $M \omega$, the number of numerical cycles from the middle of the hybridization region through the peak amplitude, and the post-Newtonian Taylor-approximant(s) used for hybridization. All pN approximants include terms up to $3.5 \mathrm{pN}$-order, see the Appendix. 
configurations modeling low-eccentricity inspiral, the mass ratio $q=m_{1} / m_{2}$ ranges from 1 to 10 , and the simulations cover a range of non-precessing spin configurations.

The initial frequency $\omega$ of the $(\ell, m)=(2,2)$ mode for the numerical waveforms ranges from $0.035 / M$ to $0.078 / M$, where $M$ denotes the sum of the initial blackhole masses, with both mean and median values of $0.048 / M$. Table 1 lists a few key parameters that distinguish the waveforms, and introduces short tags for the different contributors:

(i) Two groups use versions of the BAM code, "BAM" labels the Cardiff-Jena-PalmaVienna collaboration $[32,33,36,37,54]$, and "FAU" the contribution from the Florida Atlantic group [32, 36, 54,61].

(ii) LazEv is the RIT code $[58,62,63]$.

(iii) LEAN has been developed by Ulrich Sperhake $[55,56]$.

(iv) Two contributions use the Llama code $[40,41]$. Llama-AEI is the contribution of the AEI group [40,41], LLama-PC is the Palma-Caltech contribution [39].

(v) GATech is the Georgia Tech group, using the MayaKranc code $[48,64]$.

(vi) SpEC for the Cornell-Caltech-CITA collaboration code [24, 27-29],

(vii) UIUC stands for the University of Illinois at Urbana-Champaign team [65-67].

The numerical codes follow either of two approaches to solving the Einstein equations (see [68] for a review). The SpEC code employs the generalized harmonic formulation (see e.g. Refs. $[69,70]$ ) with gauge conditions adapted to for black hole binaries $[25,27,29]$. SpEC employs black hole excision to remove singularities in the interiors of the black holes from the computational domain. SpEC's initial-data (also using black hole excision) [71-73] is constructed with a pseudo-spectral elliptic solver [23].

All other codes use the BSSNOK formulation of the Einstein evolution equations [74-76] with hyperbolic evolution equations for the lapse and shift in the moving punctures formalism $[58,77]$. The $1+\log$ slicing condition for the lapse function [78] is "singularity-avoiding": the time slices freeze in before reaching the singularity in the black hole. This makes it possible to avoid the use of black hole excision techniques [79-82], when evolving the shift vector field $\beta^{i}$ according to the $\tilde{\Gamma}$-driver condition $[83,84]$ (extended to the moving puncture approach which allows for some free parameters which groups tune individually).

A significant amount of computational infrastructure is shared between a number of codes. With the exception of the two groups using BAM, all other moving-puncture codes are based on the Cactus computational toolkit $[85,86]$, the Carpet meshrefinement code $[87,88]$ or the EinsteinToolkit infrastructure $[89,90]$. The Cactusbased codes also use the same apparent horizon finder code (AHFINDERDiRECT) [91]. The codes Llama, LazEv, Lean and MayaKranc all use the same pseudospectral solver for the Einstein constraint equations [92], and BAM uses a variant thereof [32].

We will only very briefly summarize the main features of the numerical methods and codes, as such information is generally available elsewhere (see references above and the NINJA-1 paper [10]). There are two important exceptions: Two groups use the new Llama code, which is based on a multipatch decomposition of the numerical grid, and uses spherical coordinates in the outer zones of the grid, similar to the SpEC code. This allows a more efficient treatment of the wave zone, and to causally disconnect the outer boundaries from the wave extraction; in addition Llama uses characteristic extraction to extract the waves directly at null infinity [93,94]. The 
other important new development is the inclusion of two configurations with BH Kerr parameters 0.95 and $0.97[22,26]$, which was made possible by using superposed KerrSchild initial data $[73,95]$ in contributions from the SpEC collaboration. Suitably conformally curved initial data allows $\mathrm{BH}$ spins beyond the Kerr parameter of $\approx 0.93$, which is the maximum attainable using conformally flat initial data ([73] and the references therein). For further details on all the numerical codes used, we refer to the code references listed above, and the recent overview papers on the numerical solution of the binary black hole problem [68, 96, 97].

\subsection{Data format of contributions}

All contributions followed the format specified in Ref. [60]. The data format consists of metadata files and data files with spherical harmonic modes. The metadata specify the physical parameters of the $\mathrm{BH}$ binaries, such as mass ratio and spins, the initial frequency of the $(\ell, m)=(2,2)$ spherical-harmonic mode, eccentricity, and also authors, bibliographical references, as well as numerial methods used. This metadata format has been significantly extended since the first NINJA project to contain more information about the numerical simulations. For NINJA-1, the waveform data were stored as 3-column ASCII tables, listing the time at equidistant steps, and real and imaginary parts of the strain. For the long hybrid waveforms in NINJA-2, this format is not efficient; rather we store the time, amplitude and phase of the modes. The amplitude and phase as functions of time exhibit much less temporal structure than the complex waveform's oscillatory behavior. Therefore, amplitude and phase at arbitrary time can easily be recovered by interpolation from a drastically reduced number of time steps. Consequently, data may be provided with unequal time-spacing with only as many steps as required to accurately regenerate the original hybrid waveform with simple linear interpolation.

\subsection{Initial data and eccentricity}

Specifying initial data for black hole binaries in a non-eccentric inspiral is by itself a non-trivial problem. The elliptic constraint equations of general relativity need to be solved numerically, and the free data, which serve as input to these equations and select a specific configuration of black holes, have to be chosen in a judicious way (for a general overview see, e.g., Ref. [98]).

The moving puncture and generalized harmonic codes differ in the way they specify the free data for the constraint equations, and correspondingly in how they encode the black hole parameters. The codes based on the "moving puncture" approach use puncture initial data [99-102] to model black holes, resulting in initial data that contain a separate asymptotically flat end within each black hole. The lapse and shift fields, which determine the coordinate gauge, are initially set to trivial values, and quickly pick up values that keep the geometry of the black holes smooth and almost time independent. The SpEC code uses quasi-equilibrium excision initial data where the interior of the black-hole horizons has been excised from the numerical grid $[71,72,103]$. The constraint equations are solved using the conformalthin-sandwich formulation of the initial-value problem $[104,105]$. These data also specify an initial lapse and shift, thus the evolutions can already be started in an appropriate coordinate gauge.

All of the codes solve the elliptic constraints with pseudo-spectral numerical 
codes $[23,92]$, sharing numerical infrastructure as noted above. Initial data always correspond to the center-of-mass rest frame, such that the net linear momentum vanishes initially. All codes take input parameters that determine the individual black-hole masses $m_{i}$, spins $\vec{S}_{i}$, momenta $\vec{P}_{i}$ and coordinate separation $D$ of the black holes. We note however that in the dynamical strong field regime of general relativity, definitions of mass, spin, and linear momentum of individual black holes are ambiguous. In our case, initial separations of the black holes are large enough to match with post-Newtonian waveforms to construct hybrids, and such ambiguities are in some sense hidden in this matching procedure and associated errors.

In order to achieve non-eccentric inspiral, appropriate input parameters have to be found, i.e., appropriate momenta have to be chosen for given masses, spins, and separation. No precise definition of eccentricity is available in general relativity, and one therefore usually resorts to quantities inspired from Newtonian gravity, which quantify those oscillations in the black hole tracks or wave signal that are associated with eccentricity, see e.g., the discussion in Ref. [106]. The initial parameters are determined by a number of different methods, using either an initial guess from a standard post-Newtonian or effective-one-body (EOB) approximation based on Refs. [107-109], or adding an iterative procedure to further reduce the eccentricity, based on Refs. $[29,50,110,111]$. Measured orbital eccentricities are listed in Table 1, and have been checked for consistency by an independent estimate of the eccentricity based on the submitted GW information.

\subsection{Gravitational-wave extraction}

The gravitational-wave signal can only be defined unambiguously at null infinity. The first code that is capable of computing the wave signal at null infinity for black hole coalescences has become available since the NINJA-1 project, and is based on combining a characteristic extraction method with the Llama code [40,41], and several waveforms have been contributed using this code. Also, these calculations have provided rigorous error estimates for procedures where the wave signal is extracted at finite radius, or at a sequence of radii, combined with an extrapolation to infinite extraction radius [112], thus providing justification to such approximations.

Information about extraction radius were included for most contributions. The extraction radii ranged from $75 M$ to $500 M$, with a median of $90 M$. For four contributions the GW signal was read off at null infinity using characteristic extraction [40,113], roughly ten contributions extrapolated the GW signal to infinity from a number of extraction radii. Two techniques are used to extract an approximate gravitational waveform at finite extraction radius: SpEC uses the Regge-WheelerZerilli method [112,114-117] to compute the strain directly; all other contributions use the Newman-Penrose curvature scalar $\psi_{4}$ [118]. Computation of the strain from $\psi_{4}$ requires two time integrations, which requires the proper choice of the constants of integration, and may require further "cleaning procedures" to get rid of artifacts resulting from the finite extraction radii, as discussed in detail in Ref. [119]. Several techniques were used in practice, based on time domain [120], or frequency domain methods such as [119], or heuristic methods of suppressing low frequency Fourier modes, or combining the latter with the method of [120] as in the BAM submissions. 


\subsection{Numerical Methods, accuracy, and sources of error}

The numerical algorithms employed in the various codes agree in many details: For the time discretization, all codes use fourth or fifth order accurate explicit algorithms based on the method of lines. The consistent experience of different groups is that finite difference errors are dominated by the spatial discretization, correspondingly all the moving puncture codes use at least sixth order finite differencing for the spatial discretization. The SpEC code uses a multi-domain pseudospectral method with a large number of domains, which shows exponential convergence.

The moving puncture codes use a simple hierarchy of fixed refinement boxes which follow the motion of the black holes, and information between the boxes is communicated using buffer zones and a variant of Berger-Oliger mesh-refinement. Interpolation between refinement levels is performed with spatial polynomial interpolation of fifth (Llama, UIUC, LazEv, Lean and MayaKranc) or sixth order (BAM). Time interpolation at mesh-refinement boundaries introduces second-order errors, which does however not appear to dominate numerical errors.

\section{PN-NR Hybrid Waveforms}

While post-Newtonian $(\mathrm{pN})$ methods accurately approximate GW signals throughout early inspiral, they become increasingly unreliable towards late inspiral and merger [121]. Numerical relativity (NR) simulations are capable of accurately computing inspiral, merger, and ringdown portions of binary coalescence $[58,77,96$, 122], but are too computationally expensive to extend far into the inspiral regime [27]. Hybrid waveforms are the result of smoothly blending together $\mathrm{pN}$ and NR waveforms to form a waveform that covers the full BBH dynamics. For the NINJA-2 project, each NR group has produced their own hybrid waveform after ensuring that the $\mathrm{pN}$ portions all agree. We expect some systematic errors resulting from errors in the $\mathrm{pN}$ approximation, the choice of blending region, and the hybridization method $[16,18$ $20,27,123,124]$. The NINJA-2 hybrid waveforms do not contain effective-one-body extensions of $\mathrm{pN}$ approximants, although these have also been used to model complete waveforms (e.g. $[19,125,126])$.

Hybridization uses least-squares fits to determine the extrinsic parameters for the $\mathrm{pN}$ waveform $[124,127,128]$. In general, this is accomplished by evaluating

$$
\delta(\vec{u}, a)=\min _{\{\vec{u}, a\}} \int_{s_{2}}^{s_{1}}\left|\Upsilon_{\mathrm{pN}}(s, \vec{u})-a \Upsilon_{\mathrm{NR}}\left(s, \vec{u}_{0}\right)\right|^{2} \mathrm{~d} s
$$

where $\Upsilon$ represents waveform data relating to strain [e.g., $h(t)=h_{+}(t)-i h_{\times}(t)$, $\arg [h(t)]$ or $\tilde{h}(f)]$. If $\Upsilon$ is derived from the time domain, then $s=t$; if $\Upsilon$ is in the frequency domain, then $s=f$. For either case, $\left[s_{1}, s_{2}\right]$, chosen within the domain of both the $\mathrm{pN}$ and $\mathrm{NR}$ data sets, defines the integration interval and, in most cases, the blending region. The vector $\vec{u}$ denotes the set of $\mathrm{pN}$-parameters over which the fitting is performed. For example, $\vec{u}=\left(t_{\text {shift }}, \phi_{\text {shift }}, \mu\right)$ corresponds to adjusting time- and phase- shift and the mass ratio of the $\mathrm{pN}$ waveform to match the NR waveform. The best-fit parameters are denoted by $\vec{u}^{*}$. The amplitude scaling factor, $a$, is often fixed to $a=1$, but may be included in the fitting parameters [127]. Finally, in the limit $s_{1} \rightarrow s_{2}$, this procedure reduces to enforcing equality of $\Upsilon_{\mathrm{pM}}$ and $\Upsilon_{\mathrm{NR}}$ at $s_{1}=s_{2}$, as well as equality of the first derivative.

More explicitly, hybridization may be performed via the following algorithm: 
(i) Choose $\left[s_{1}, s_{2}\right]$ within the $\mathrm{pN}$ and $\mathrm{NR}$ data sets. Ideally, $\left[s_{1}, s_{2}\right]$ is sufficiently early so that both $\mathrm{pN}$ and NR sets should be accurate.

(ii) Evaluate Eq. (1); apply $\left\{\vec{u}^{*}, a^{*}\right\}$ to the $\mathrm{pN}$ data set, resulting in $\Upsilon_{\mathrm{pN}}^{*}$. Measure error quantities relating to fit.

(iii) If desired, adjust $\left[s_{1}, s_{2}\right]$ and iterate (i) and (ii), to find a preferred interval $\left[s_{1}^{*}, s_{2}^{*}\right]$.

(iv) Defining a monotonic function $z(s)$ such that $z\left(s<s_{a}\right)=0$ and $z\left(s>s_{b}\right)=1$, the hybrid is given by

$$
\Upsilon_{\mathrm{Hyb}}(s)=[1-z(s)] \Upsilon_{\mathrm{pN}}^{*}+a^{*} z(s) \Upsilon_{\mathrm{NR}}
$$

Note that the transition region $\left[s_{a}, s_{b}\right]$ is generally taken to be a sub-interval of $\left[s_{1}^{*}, s_{2}^{*}\right]$, sometimes consisting of a single point.

This general formalism leaves many decisions open, and the following specific choices were made during hybridization:

- For the SpEC waveforms the integrand of Eq. (2) was taken to be the square of the phase-difference only [128], $\Upsilon=\arg [h(t)]$, and maximation was performed over $t_{\text {shift }}$ and $\phi_{\text {shift }}$ only, without any adjustments to the amplitude $\left(a^{*} \equiv 1\right)$. The time-interval $\left[t_{1}, t_{2}\right]$ was chosen to correspond to the frequencies listed in Table 1 , without any iterative adjustments of $t_{1}$ and $t_{2}$.

- The RIT waveforms similarly use $\Upsilon=\arg [h(t)]$ for Eq. (1), but employ the limit as $t_{1} \rightarrow t_{2}$ to determine $\vec{u}=\left(t_{\text {shift }}, \phi_{\text {shift }}\right)$ at $M \omega=0.075$. Then, the transition function is $z_{\mathrm{RIT}}(t)=x(t)^{3}\left[6 x(t)^{2}-15 x(t)+10\right]$, where $x(t):=\left(t-t_{1}\right) /\left(t_{2}-t_{1}\right)$ for a finite interval $\left(t_{1} \neq t_{2}\right)$, guaranteeing $C^{2}$ behavior at $t=t_{1}$ and $t_{2}$ [129].

- For the Lean waveforms hybridization is performed in a similar way [130], using the transition function $z(t)=70 x^{9}-315 x^{8}+540 x^{7}-420 x^{6}+126 x^{5}$, and individual mode amplitudes of the PN waveforms are rescaled such that their average over the matching window agrees with the numerical result.

- The UIUC waveforms use $\Upsilon=h(t), a=1$, using as free parameters the initial PN phase and orbital angular frequency. Equation (2) is then used to construct the final hybrid, with $z(t)=\left(t-t_{1}\right) /\left(t_{2}-t_{1}\right)$ as in [131]. The time-interval $\left[t_{1}, t_{2}\right]$ was chosen to correspond to the UIUC frequencies listed in Table 1, without any iterative adjustments of $t_{1}$ and $t_{2}$.

- The GATech hybridization follows [128] and is done in the time domain with $\Upsilon=h(t)$ and $\vec{u}=\left(t_{\text {shift }}, \phi_{\text {shift }}\right)$. Equation (1) is evaluated over $\{\vec{u}, a\}$ and then equation (2) is used to construct the final hybrid, with $z(t)=\left(t-t_{1}\right) /\left(t_{2}-t_{1}\right)$. The fitting intervals are given in Table 1 .

Figure 3 and 4 show exemplary plots of the resulting hybrid waveforms. For aligned spins, orbital hangup extends the inspiral to smaller separation and higher frequencies. This is apparent in Fig. 3 in that the last ten GW cycles (as indicated by the small circles) take less time, and in Fig. 4 by the shift toward higher frequencies.

\section{Validation and comparison of hybrid waveforms}

Each NR group verified that their waveforms met the minimum NINJA-2 requirements before submission, as described in Sec. 1. Once submitted, a series of checks were performed in order to validate the waveforms against each other. In the first stage the post-Newtonian expressions and codes were compared against each other and the literature. This resulted in a set of codes in various languages producing waveforms that agree well in both phase and amplitude (see Appendix A). 

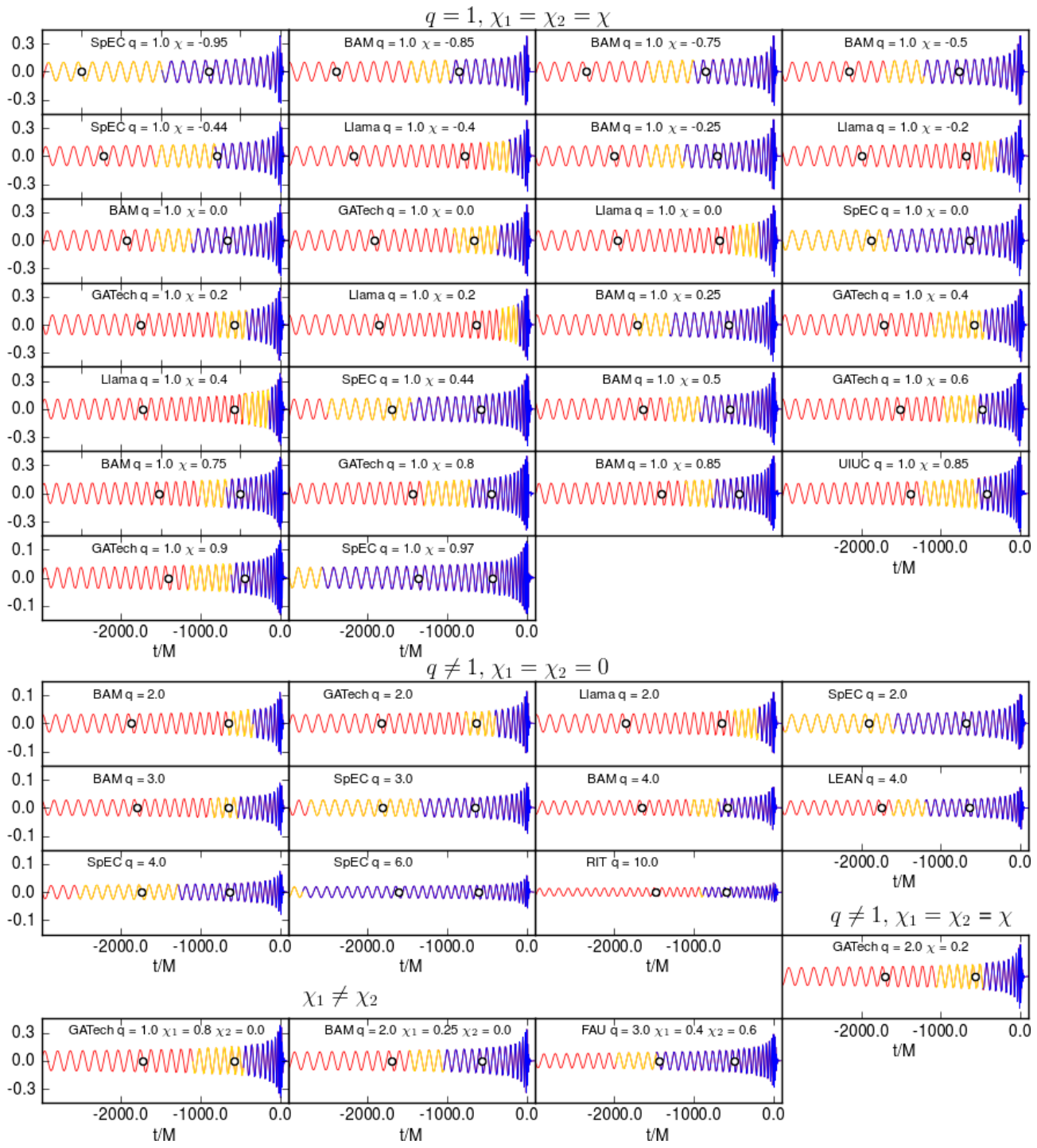

Figure 3. Summary of all submitted hybridized waveforms $(\mathbf{r} / \mathbf{M}) \mathbf{h}_{+}$The $\mathrm{x}$-axis shows time in units of $\mathrm{M}$ and the $\mathrm{y}$-axis shows the real part of the $(\ell, m)=$ $(2,2)$ component of the dimensionless wave strain $(r / M) h=(r / M)\left(h_{+}-i h_{\times}\right)$. The top group shows equal-mass equal-spin waveforms. The middle group shows unequal-mass and zero-spin waveforms, and the bottom group show unequal spin waveforms. The black circles indicate 10 and $20 \mathrm{GW}$ cycles measured from the waveform peak. The hybridization frequency range is shown in the yellow line. The post-Newtonian part is shown in red line and the NR portion occurring after hybridization is shown in blue line. 


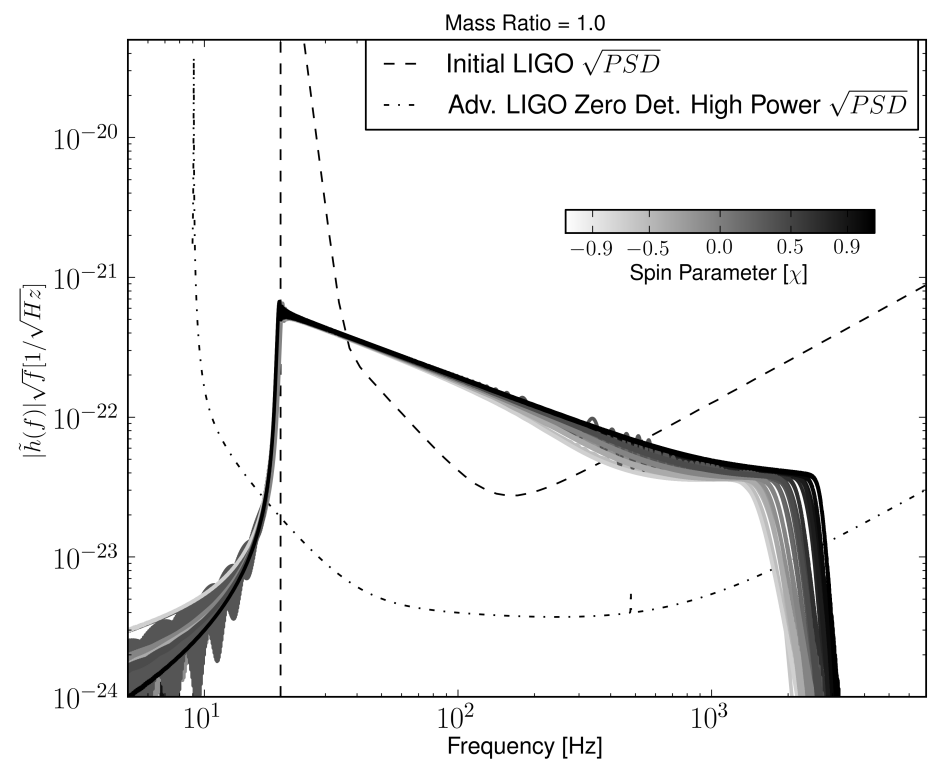

Figure 4. Sample frequency domain plot Shown are the plots of $|\tilde{h}(f)| \sqrt{f}$ of $(\ell, m)=(2,2)$ mode for all equal-mass equal-spin waveforms. The waveforms are scaled to $10 \mathrm{M}_{\odot}$ and are placed at $100 \mathrm{Mpc}$. The Fourier transforms show monotonic behavior in the spin parameter $\chi=\left(q \chi_{1}+\chi_{2}\right) /(q+1)$ highlighting the orbital hang-up effect due to spin. The vertical line indicates $20 \mathrm{~Hz}$, the required upper bound on the initial frequency of the hybrids.

\subsection{Time-domain and frequency-domain checks}

In the second stage of validation we examined the $(\ell, m)=(2,2)$ mode of the hybrid waveforms. We first plotted the last 40 cycles of each waveform - enough to include the full NR portion, the hybridization region, and some of the $\mathrm{pN}$ portion - and looked for any anomalies such as "kinks" caused by the hybridization procedures. Similar visual checks were performed on the amplitudes of the Fourier transforms of the entire waveforms. This process identified a few issues, including a bug in one hybridization code, which were then corrected. While this was useful, it was only possible due to the relatively small number of waveforms in NINJA-2 and the fact that only the $(\ell, m)=(2,2)$ mode was examined. For future NINJA projects it will be necessary to automate this process, the NINJA-2 data analysis may suggest methods for such automation.

\subsection{Overlap Comparisons}

In this check the waveforms were compared against each other using matched-filtering techniques. The inner product between two real waveforms $s_{1}(t)$ and $s_{2}(t)$ is defined as

$$
\left(s_{1} \mid s_{2}\right)=4 \Re \int_{0}^{\infty} d f \frac{\tilde{s}_{1}(f) \tilde{s}_{2}^{\star}(f)}{S_{n}(f)}
$$



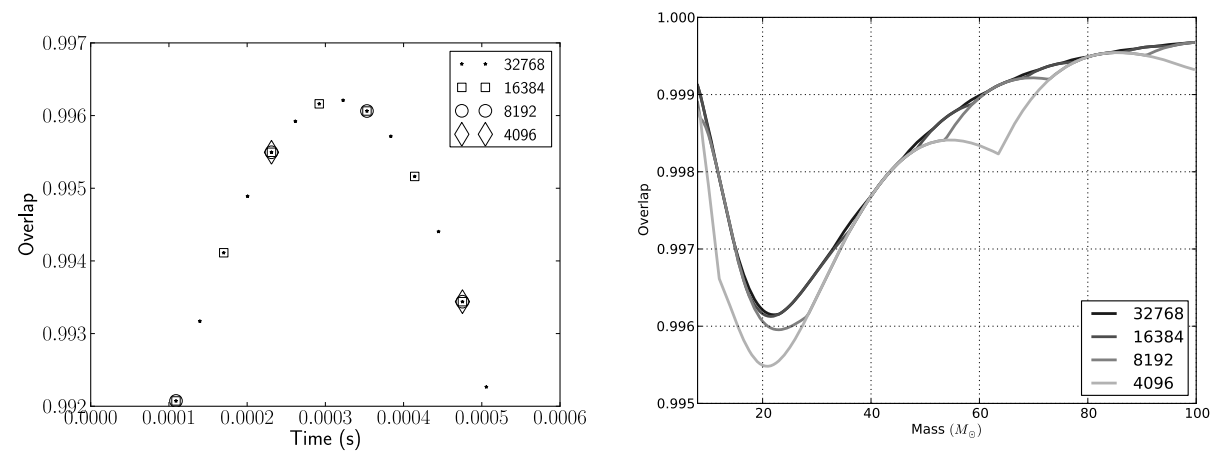

Figure 5. Left: Overlap time series between the GATech and SpEC equal-mass, non-spinning waveforms scaled to $20 M_{\odot}$ at different sample rates. At $4096 \mathrm{~Hz}$ the sample point nearest the maximum is sufficiently far that the overlap is significantly underestimated, where we are interested in differences to one part in $10^{-5}$. Right: Overlaps between the same waveforms as a function of mass, at different sample rates. All overlaps are calculated against the early aLIGO noise curve.

where $S_{n}(f)$ is the power spectral density (see Fig. 1). The overlap is then obtained by normalization and maximization over relative time and phase shifts, $\Delta t$ and $\Delta \phi$.

$$
\left\langle s_{1} \mid s_{2}\right\rangle:=\max _{\Delta t, \Delta \phi} \frac{\left(s_{1} \mid s_{2}\right)}{\sqrt{\left(s_{1} \mid s_{1}\right)\left(s_{2} \mid s_{2}\right)}} .
$$

There is an important subtlety involved in calculating these overlaps. Usually, all possible time-shifts are evaluated simultaneously by a suitable combination of fast Fourier transforms. This results in overlaps at time-shifts at discrete values of $\Delta t$ spaced by the inverse sampling frequency. The result of time-maximization is then taken to be the maximum of this discrete time-series. When comparing two very similar waveforms, such as two NR simulations of the same system, the overlap function becomes very sharply peaked. In units where $G=c=1,1 M_{\odot}=4.93 \times 10^{-6} s$, and in these units time shifts of well below $1 M$ can lead to significant changes in the overlap. It is therefore imperative that the sample rate used in calculating the overlap be large enough to find the true maximum.

This issue is demonstrated in Fig. 5. The left panel shows the overlap function resulting from the comparison of two equal-mass, non-spinning waveforms sampled at four different rates. At $4096 \mathrm{~Hz}$ the peak of the function is missed and the overlap is underestimated. The consequence of this is illustrated on the right of Fig. 5 which shows that the overlap as a function of mass exhibits oscillations. As the mass changes, the phase-shift $\Delta \phi$ that maximizes Eq. (3) also changes. For some masses, the optimal 
phase-shift will occur at one of the discretely sampled time-shifts, and the results will be correct. For other masses, the true extremum will occur between discretely sampled time-shifts (as for $4096 \mathrm{~Hz}$ in the left panel), and the computed overlap will be erroneously too low. Henceforth in this paper, all overlaps are calculated at $32768 \mathrm{~Hz}$. The LIGO/Virgo matched filter searches operate on data at $4096 \mathrm{~Hz}$, however this issue is not a problem in these searches for reasons that can be seen in these two images. The loss of overlap by undersampling is no larger than $0.2 \%$. The search utilizes a bank of templates which discretizes the parameter space. In constructing the template bank we have already incurred a potential loss of SNR of $3 \%$, and this effect is therefore negligible.

Before discussing overlaps between the submitted NINJA hybrid waveforms, we first present comparisons between time-domain post-Newtonian waveforms of the kind used to construct hybrid waveforms. (See the Appendix for a summary of the $\mathrm{pN}$ approximants used.) These waveforms terminate at $M \omega=0.136,0.114,0.069$, and 0.135 for $\mathrm{T} 1, \mathrm{~T} 2, \mathrm{~T} 3$ and $\mathrm{T} 4$ respectively. At $M=10 M_{\odot}$ these correspond to termination frequencies of $435 \mathrm{~Hz}, 369 \mathrm{~Hz}, 222 \mathrm{~Hz}$ and $439 \mathrm{~Hz}$, respectively. The results of our overlap calculations are shown in Fig. 6. Ref. [132] contains a far more extensive comparison between post-Newtonian approximants, although that analysis differs in several important aspects from what has been done here, notably the overlaps are maximized over the intrinsic mass parameters of one waveform. However the qualitative conclusion that T1 matches better with T2 and T4 than with T3 is consistent with our results. These results should be borne in mind when evaluating overlaps between hybrid waveforms using different $\mathrm{pN}$ approximants. In particular, overlaps at lower masses will be dominated by the influence of the two $\mathrm{pN}$ waverforms, and therefore hybrid waveforms using $\mathrm{T} 3$ will have relatively low matches against hybrid waveforms using T1. Note also that T3 and T4 diverge from T1 as mass increases, this is precisely why we need to transition to numerical-relativity waveforms.

Let us now discuss the main results of this section, the overlap between different submitted hybrid waveforms. For six of the black hole configurations listed in Table 1, hybrids have been constructed independently for different numerical waveforms, and overlaps were computed between each pair of waveforms in a group. Below we are showing overlap plots for all six cases: The $q=\{1,2\}$ non-spinning waveform submissions are shown in Fig. 7, $q=\{3,4\}$ non-spinning waveforms in Fig. 8, and $q=1, \chi_{i}=0.4,0.85$ spinning waveforms in Fig. 9. We will discuss the $q=\{1,2\}$ non-spinning cases in more detail.

At the high-mass end, where the NR portion of the waveform dominates the overlap, the overlaps approach 1 . Since all these waveforms model the same physical system, this is the expected behavior. High overlaps at high masses indicate good agreement between different NR codes, and the results here are consistent with the detailed study of the $q=1$ case was performed in the Samurai project [21]. At the low-mass end, where the overlap is dominated by the $\mathrm{pN}$ portion of the waveform, the behavior is qualitatively as expected from Fig. 6 . In particular the overlap between the BAM hybrid using T1 and the SpEC hybrid using T4 is lower than the matches between the other hybrids, all of which use T4. In the region between $\sim 15-30 \mathrm{M}_{\odot}$ there are drops in the matches between hybrids using T4. In this mass range the hybridization regions are passing through the most sensitive frequency band, and the mismatches are due in part to different choices of hybridization methods and parameters. The question of how many NR cycles are needed in order to produce a robust hybrid waveform is an area of active research [16-20]. 

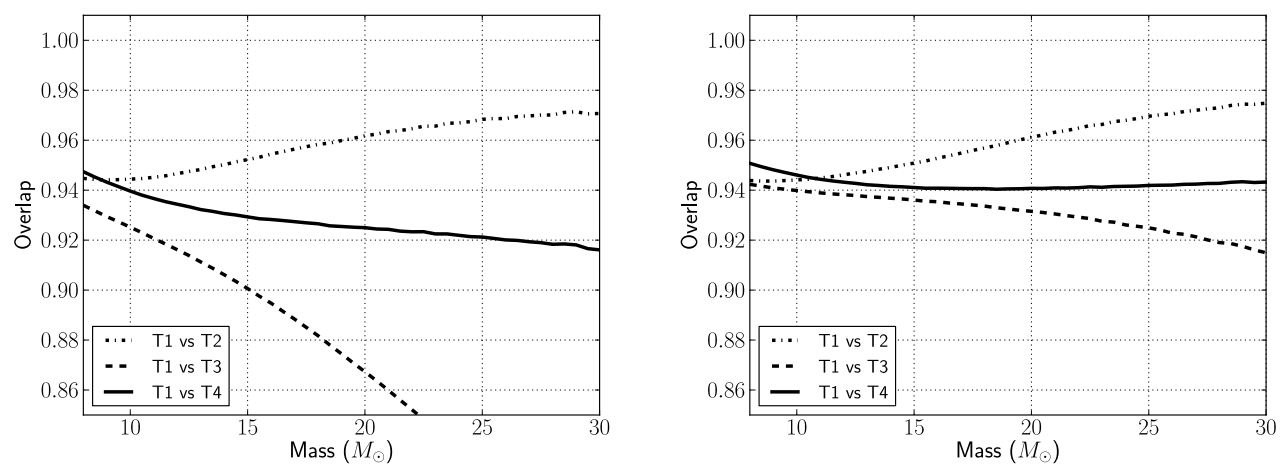

Figure 6. Overlaps between equal-mass, non-spinning, post-Newtonian timedomain waveforms. Note in particular the discrepancy between T1 and T4, as these were used in the majority of the hybrid waveforms. On the left overlaps are calculated against the early aLIGO noise curve, on the right overlaps are calculated against the Zero-detuned, high-power noise curve. All waveforms include terms up to $3.5 \mathrm{pN}$-order, as do the post-Newtonian portions of the hybrid waveforms.

If the approximants are the same, then the mismatches will depend only on the differences in (1) the hybridization methods, (2) the hybridization frequencies (and windows), and (3) the NR data. We have performed tests to verify that the overlap due to these effects is very small. We have made overlap calculations using a whitenoise PSD, integrated between $10 \mathrm{~Hz}$ and $100 \mathrm{~Hz}$, so the hybridisation region can pass fully into and out of band as the mass is varied between $10 M_{\odot}$ and $100 M_{\odot}$, and found that the maximum mismatch due to hybridization is $0.05 \%$ for the GATech and SpEC equal-mass nonspinning hybrids. This suggests that the contribution of the hybridization to the mismatch is very small, which is consistent with the results in [16]. Mismatches for masses $M \gtrsim 150 M_{\odot}$ will be due only to differences between the numerical data, and we find these to be $0.1 \%$, which is consistent with the results for equal-mass nonspinning binaries in [21] and for $q=2$ nonspinning binaries in [124].

The overlap plots discussed thus far do not yet address the accuracy required for detection of gravitational waves. Broadly, in order to claim a detection a signal must match well against at least one template waveform used in a search. Several methods of assessing detection accuracy have been proposed [133,134], however here we take the simple aproach that the waveforms are sufficient for further detection studies if the overlap is above the the standard 0.97 threshold for a loss of no more than $10 \%$ of signals in a search. In some cases above the overlaps are not above this threshold, but 

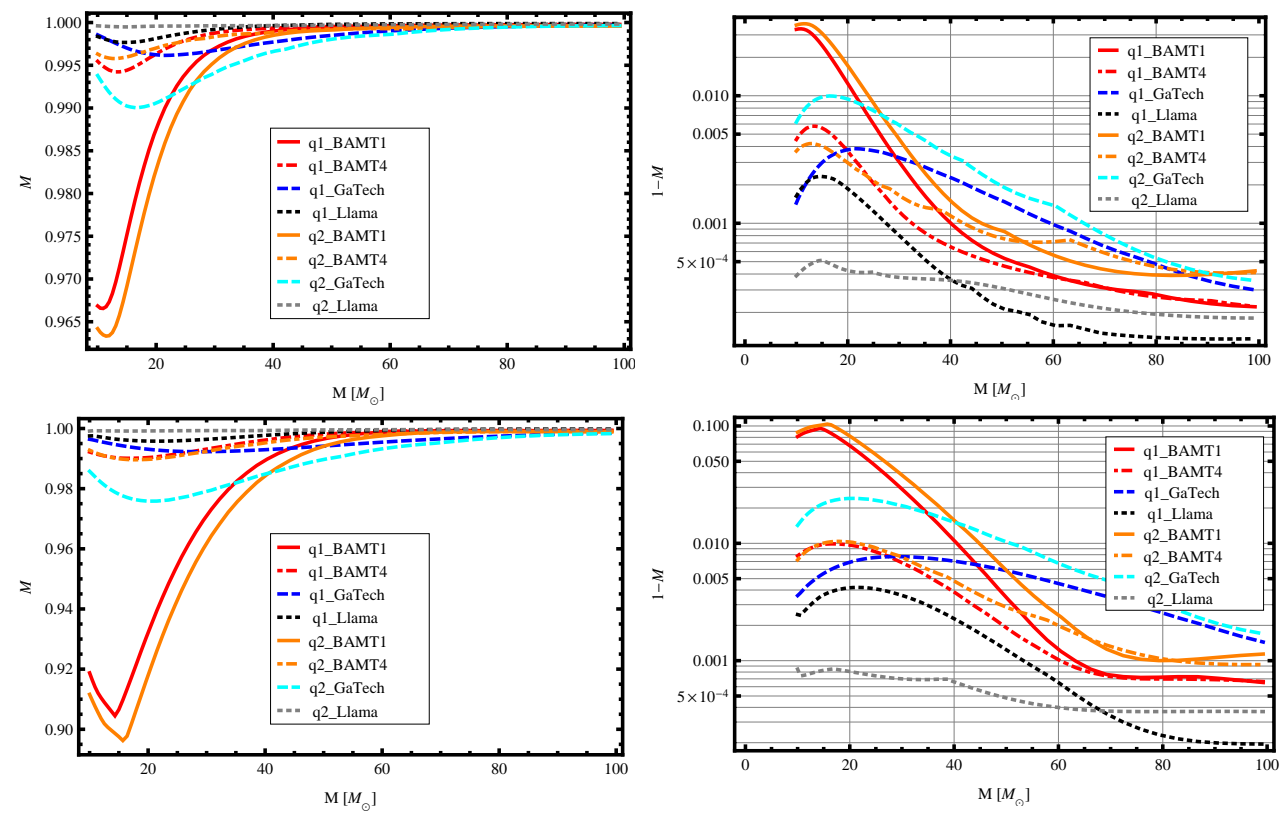

Figure 7. Differences between hybrid-waveforms for the same configuration. Plotted are the overlaps of the SpEC (TaylorT4) hybrid against the four other submissions: All plots show results for both equal mass and mass ratio two hybrid waveforms with zero spin. On the top row overlaps were computed using the early aLIGO noise curve. On the bottom row the ZeroDetuned, High-Power noise curve was used. The overlaps are above 0.98 for identical pN-approximants. For different $\mathrm{pN}$-approximants overlaps are above 0.98 for the early noise curve and above 0.90 for the Zero-Detuned, High-Power noise curve.

the appropriate quantity to evaluate to address this question is the overlap maximized over all of the physical parameters in a search. We now extend these overlap studies by maximizing over one physical parameter, the mass of one of the waveforms, as well as the time and phase. If the overlaps are now all over 0.97 (which they are), then they are acceptable for use in search-related studies.

These extended overlaps also provide insight into the "parameter estimation question," as the error in parameter recovery between two hybrid waveforms gives a rough lower bound on the errors we may expect in recovering parameters of hybrid injections with search templates. In practice parameter recovery is likely to be worse than this, as it will involve maximizing over several parameters in addition to total mass, in addition to differences between the waveforms. Example plots using the equal-mass, non-spinning MayaKranc waveform as the signal and BAM hybridized with two different $\mathrm{pN}$ approximants as the template are shown in Fig. 10. Note that in the right panel of Fig. 10 the minimum overlap is below 0.97. This minimum match will be even worse for $q>1$ binaries [16], but if maximization is done over the other physical parameters (mass ratio and spin), then the overlap will increase to well above $0.97[20]$.

At the high-mass end the overlap is dominated by NR data, and as in Fig. 7 the overlaps are high without needing to move off the signal mass. At the low-mass end the same result would be expected in a pure $\mathrm{pN} / \mathrm{pN}$ comparison although there is 

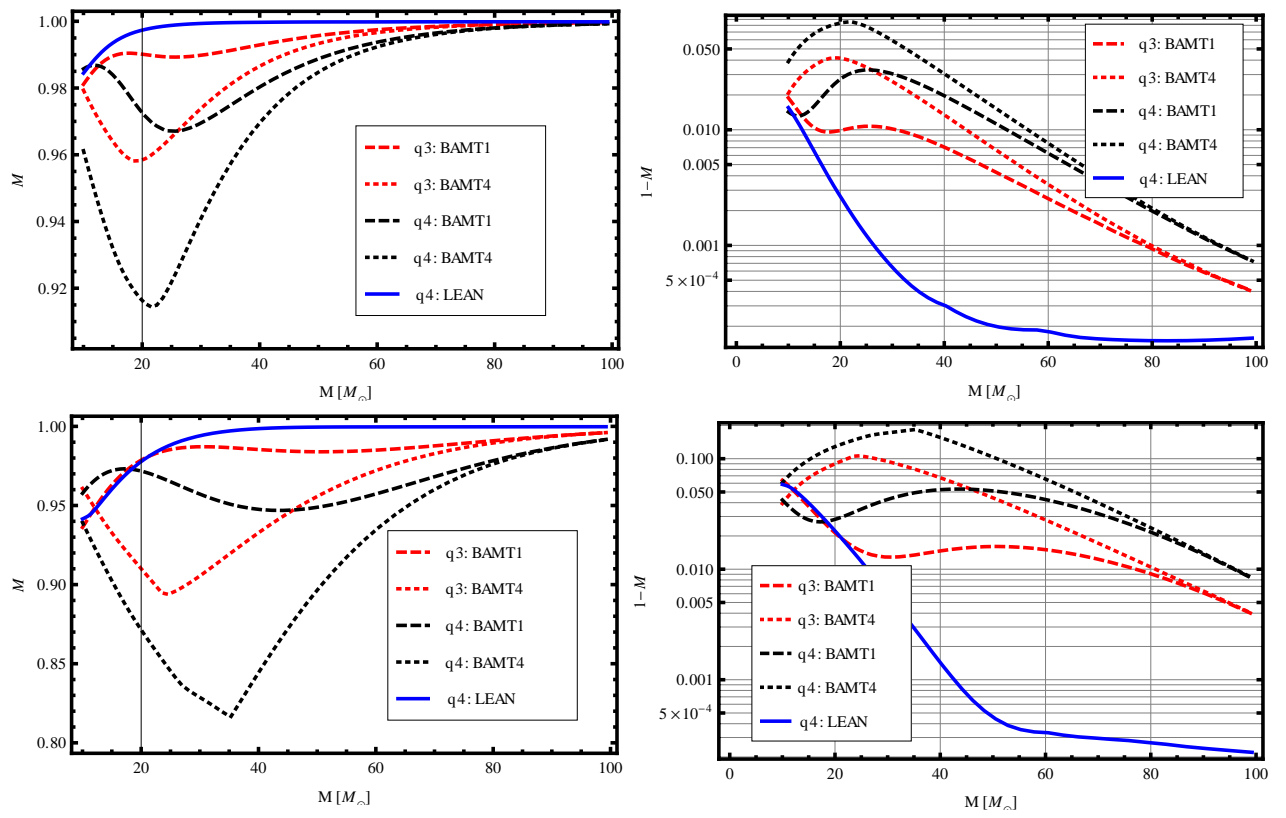

Figure 8. Differences between hybrid-waveforms for the same configuration. Plotted are the overlaps of the SpEC (TaylorT2) hybrid against the four other submissions: All plots show results for both mass ratio three and four nonspinning hybrid waveforms. On the top row overlaps were computed using the early aLIGO noise curve. On the bottom row the Zero-Detuned, High-Power noise curve was used.

enough of the hybridization in-band to reduce the overlaps. However, changing the mass introduces a phase difference that accumulates over all the cycles in-band, and so higher overlaps cannot be achieved. The result is optimal mass values close to the correct mass value, but with a lower overlap. In the middle region these factors compete. At higher masses the overlap is reduced less by changing the mass and so the recovered value of the mass can stray further from the injected value. As the hybridization passes out of band this adjustment is no longer needed. The same general behavior can be seen in comparisons between non-spinning, unequal-mass $(q=2)$ waveforms, and for equal-mass spinning waveforms, as shown in Fig. 11 which shows overlaps between waveforms with identical parameters and hybridized with the same $\mathrm{pN}$ approximants maximized over the mass of one waveform. We can infer from these results that, although we have only made a crude estimate of the parameter bias, the accuracy of the waveforms (excluding the uncertainty in the $\mathrm{pN}$ approximants) is extremely high. There are many factors that may bias parameter estimation of real signals, including uncertainties in the detector calibration, noise in the detectors and errors in $\mathrm{pN}$ waveforms used as templates. Subsequent NINJA-2 data analysis studies will attempt to quantify the effects of these factors.

\section{Conclusion}

The efficiency of searches for GW signals from BH binaries and the accuracy with which the source parameters are estimated will depend crucially on progress in 

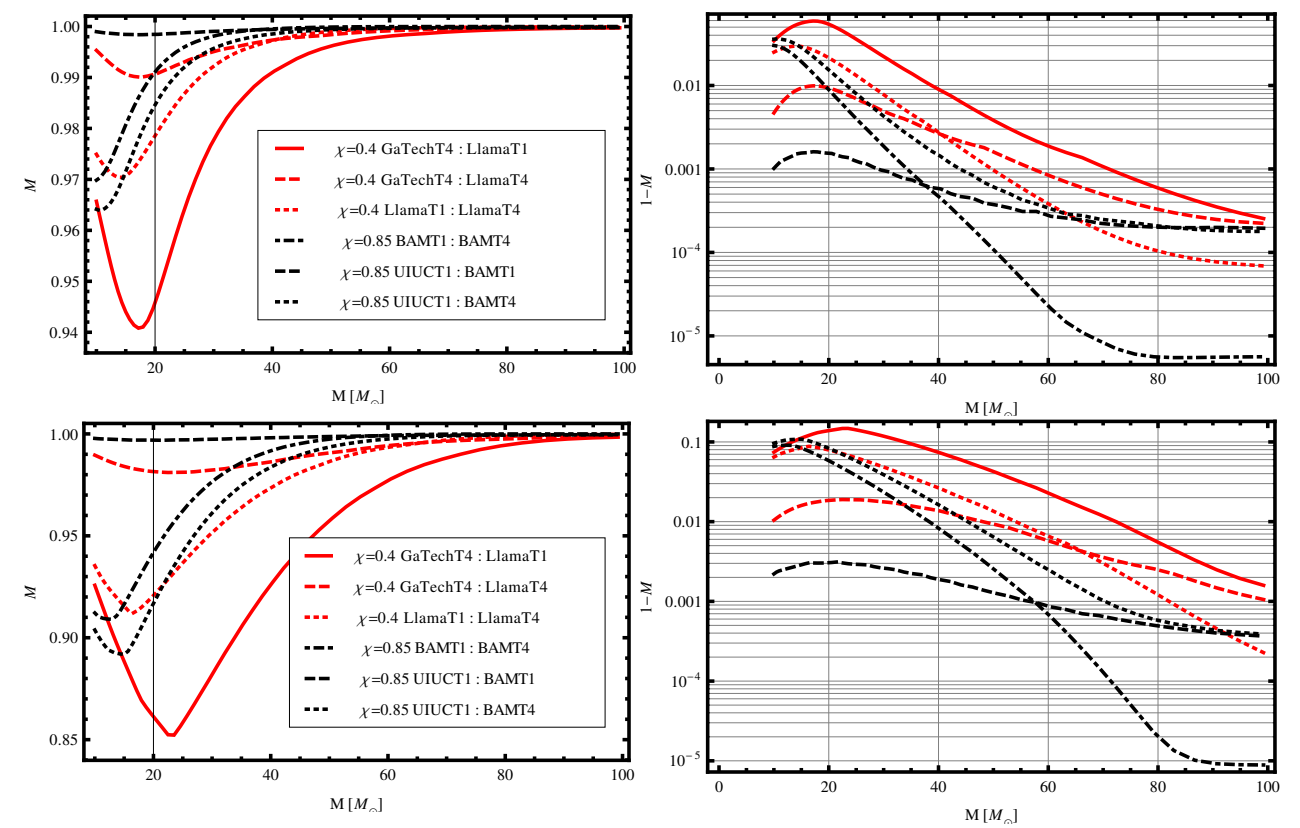

Figure 9. Differences between hybrid-waveforms for the same configuration. Plotted are the overlap comparisons of sets of equal mass spinning hybrids with $\chi_{i}=0.4,0.85$. On the top row overlaps were computed using the early aLIGO noise curve. On the bottom row the Zero-Detuned, High-Power noise curve was used. The overlaps are above 0.98 for identical $\mathrm{pN}-$ approximants. For different pN-approximants overlaps deteriorate to 0.94 for the early noise curve and above 0.85 for the Zero-Detuned, High-Power noise curve.

incorporating information from approximation methods and numerical relativity into the waveform models used in data-analysis algorithms. Even in the nonspinning case, a recent extensive comparison of different $\mathrm{pN}$ approximants [132] concludes that for masses above $12 M_{\odot}$ numerical-relativity simulations of the last orbits and merger are required for the construction of optimal detection templates. This need is expected to be even greater when spinning binaries are included. And of course, numericalrelativity simulations will be yet more important for parameter estimation.

Injections of inspiral-merger-ringdown (IMR) waveforms from analytical waveform models are already used to calibrate the analysis of detector data [135]. However, the synthesis of NR results into waveform models typically lags several years behind the most complete current sets of NR waveforms. The NINJA-2 project will consider direct injections of hybrid pN-NR waveforms, which will allow the use of waveforms which have not (yet) been used in constructing analytical models, and will avoid any additional modeling errors. Using the best available IMR waveforms will be particularly important for parameter estimation.

The first goal of the second NINJA project has been to review the submitted hybrid waveforms. In this paper we have summarized the requirements that the submitted waveforms must meet (see Sec. 1). All submitted waveforms meet these requirements, within some minor caveats that are detailed in Sec. 1. We have also demonstrated the validity of the hybrid waveforms for use in the NINJA-2 project with more detailed consistency checks of the submitted data. 

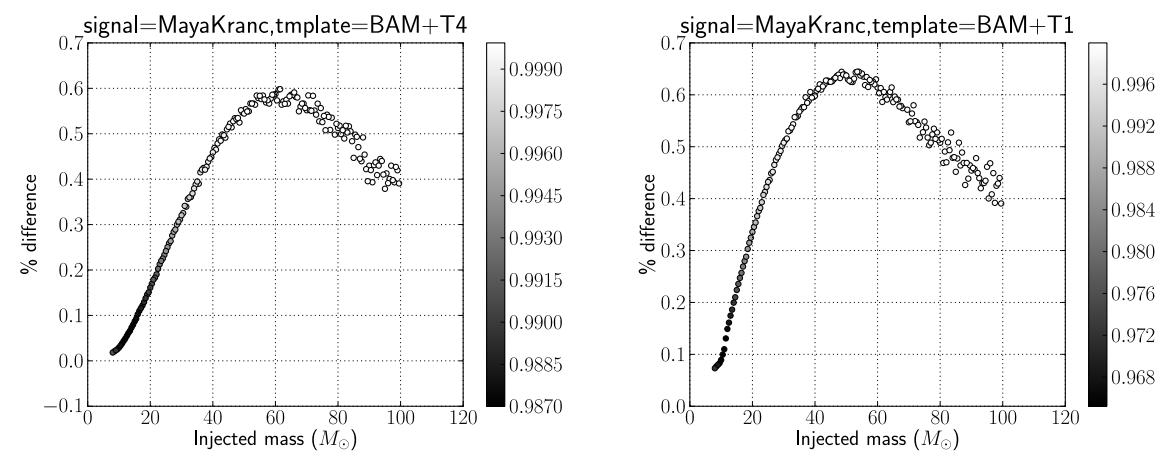

Figure 10. Overlaps and mass-bias when searching for one hybrid waveform with another hybrid waveform of the same configuration. Left panel: the $q=1$ nonspinning MayaKranc waveform is taken as the signal, and the $q=1$ non-spinning BAM waveform hybridized with TaylorT4 taken as the template. Right panel: the $q=1$, non-spinning MayaKranc waveform is taken as the signal and the $q=1$ non-spinning BAM waveform hybridized with TaylorT4 is taken as the template. In both panels, maximization is done over the mass of the template, as well as over time and phase. The horizontal axis gives the mass of the signal; the vertical axis gives the fractional difference between the injected mass and the mass of the template that maximizes the overlap. Overlaps are calculated against the early aLIGO noise curve.

The validation of and comparisons between the submissions, which we have presented here, are a major feature of NINJA-2 that was not present in NINJA-1, and will be indispensable when analyzing the results from systematic injection studies over the course of the NINJA-2 project. A total of 63 waveforms from 8 different groups were contributed to NINJA-2, corresponding to 46 distinct NR waveforms, and 29 different configurations of mass ratio and spins. For six configurations, multiple numerical waveforms were submitted, and 16 numerical waveforms were hybridized with two different $\mathrm{pN}$ approximants (TaylorT1 and TaylorT4). This has allowed waveform comparisons and tests of the accuracy standards, as discussed in detail in Sec. 5, and summarized below. For each submission, significant further information has been included in metadata files as specified in [60], e.g., hybridization frequencies, eccentricities, or literature references. This allowed us to automatically generate information such as Table 1 and has proved crucial in systematically analyzing the data set. Verifying these submissions, preparing a consistent data set, and evaluating their accuracy by comparing submissions with the same mass-ratio and spin, has been a formidable task, and in the present paper we only report on the $(\ell, m)=(2,2)$ spherical-harmonic modes. Based on our various checks and comparisons as reported 

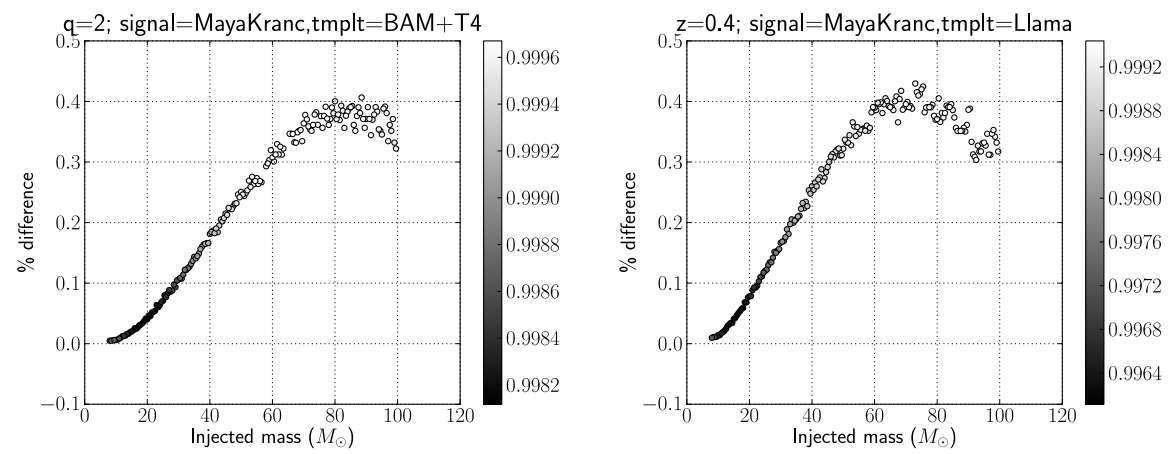

Figure 11. Overlaps and mass-bias when searching for one hybrid waveform with another hybrid waveform of the same configuration. Left panel: the $q=2$ nonspinning MayaKranc TaylorT4 waveform is taken as the signal, and the $q=2$ nonspinning BAM waveform hybridized with TaylorT4 taken as the template. Right panel: the $q=1, \chi_{1}=\chi_{2}=0.4$ MayaKranc Taylor T4 waveform is taken as signal and searched for with the Llama waveform of the same parameters and $\mathrm{pN}$ approximant. In both panels, maximization is done over the mass of the template, as well as over time and phase. The horizontal axis gives the mass of the signal; the vertical axis gives the fractional difference between the injected mass and the mass of the template that maximizes the overlap. Overlaps are calculated against the early aLIGO noise curve.

in this paper, we judge the submitted waveforms suitable for the NINJA-2 project. Parameter estimation places particular stringent demands on waveform templates. We believe the NINJA-2 waveforms to be of sufficient quality to estimate size and shape of maximum likelihood contours. However, the large truncation error of the post-Newtonian expansion in the cycles before and during hybridization will induce systematic errors; further investigations will be necessary before these waveforms can be used to check the accuracy of the estimated parameters themselfes (i.e. the location of the maximum likelihood contour, rather than its shape).

As seen in Fig. 2, the submissions primarily cover only two lines in the $(q, \chi)$ plane, leaving large regions of parameter space unexplored. We also do not consider any precessing signals, which adds several dimensions to the parameter space. These issues will be explored in future NINJA projects. Extending our analysis to nondominant spherical-harmonic modes, and to a larger volume of parameter space, will mark a significant challenge for the future, and will require a significant extension of our methods of analysis, and of automatizing them, and will constitute a substantial research project by itself.

An important shortcoming in our analysis of waveform accuracy is that it is 
currently not possible to accurately estimate the $\mathrm{pN}$ truncation error. A reasonable approach seems to be to compare $\mathrm{pN}$ approximants, which are accurate to the highest known order, but differ in terms beyond this order (3.5pN without spins). These deviations still depend strongly on the choice of $\mathrm{pN}$ approximants used in any comparison, and on the mass ratio and spins. For example, in Figs. 6, 7 and 10 we compare the use of the TaylorT1 and TaylorT4 approximants for mass ratio $q=1$ and zero spins, Fig. 7 also includes $q \neq 1$. Previous work implies that TaylorT4 performs exceptionally well in these cases $[29,35]$, apparently by coincidence, but beyond that it is not possible to make precise quantitative statements; this figure simply illustrates the general level of mismatch error that we may expect between various $\mathrm{pN}$ approximants across a range of binary masses.

We caution that the comparisons presented in Sec. 5 were done only for subset of the black hole configurations, which do not include the most extreme cases, and not the cases with unequal Kerr parameters. Further work will be necessary to establish with similar confidence that all NINJA-2 hybrid waveforms are of similar quality.

Our waveform comparisons in Sec. 5 are consistent with previous results: mismatches between waveforms are dominated by the choice of $\mathrm{pN}$ approximant, while NR and hybridization errors are far smaller. Hybridization choices and methods certainly affect overlaps, although the mismatch due to hybridization appears to be less than a fraction of a percent. This is not expected to have any noticeable effect on detection, but is likely to impact parameter estimation. The degree to which these effects will bias searches is one of the key question we hope NINJA-2 will be able to answer. In particular, theoretical studies such as the ones presented in this paper cannot replace a complete parameter estimation analysis, and it is not clear how theoretical studies based on Gaussian noise can predict the performance of GW searches in real noise. Another important goal of the NINJA-2 project is thus to build up experience in comparing simple theoretical studies with the full $\mathrm{GW}$-search plus parameter estimation pipeline exercised in actual observations of GW events.

\section{Acknowledgments}

We thank Ilya Mandel for helpful discussions.

We acknowledge gratefully support from the National Science Foundation under NSF grants PHY-1040231, PHY-0600953, PHY-0847611, AST-1028087, DRL-1136221, OCI-0832606, PHY-0903782, PHY-0929114, PHY-0969855, AST1002667, PHY-0650377, PHY-0963136, PHY-0855315, PHY-0969111, PHY-1005426, PHY-0601459, PHY-1068881, PHY-1005655, PHY-0653550, PHY-0955773, PHY0653443, PHY-0855892, PHY-0914553, PHY-0941417, PHY-0903973, PHY-0955825, NSF cooperative agreement PHY-0757058, by NASA grants 07-ATFP07-0158, NNX07AG96G, NNX10AI73G, NNX09AF96G, NNX09AF97G, by Marie Curie Grants of the 7th European Community Framework Programme FP7-PEOPLE-2011-CIG CBHEO No. 293412, by the DyBHo-256667 ERC Starting Grant, and MIRG-CT-2007205005/PHY, and Science and Technology Facilities Council grants ST/H008438/1 and ST/I001085/1. Further funding was provided by the Sherman Fairchild Foundation, NSERC of Canada, the Canada Research Chairs Program, the Canadian Institute for Advanced Research, Govern de les Illes Balears, the Ramón y Cajal Programme of the Ministry of Education and Science of Spain, contracts AYA201015709, CSD2007-00042, CSD2009-00064 and FPA2010-16495 of the Spanish Ministry of Science and Innovation, the Royal Society, and the Research Corporation for 
Science Advancement. Computations were carried out on Teragrid machines Lonestar, Ranger, Trestles and Kraken under Teragrid allocations TG-PHY060027N, TGMCA99S008, TG-PHY090095, TG-PHY100051, TG-PHY990007N, TG-PHY090003, TG-MCA08X009. Computations were also performed on the clusters "HLRB2" at LRZ Munich, "NewHorizons" at RIT (funded by NSF Grant Nos. AST1028087, DMS-0820923 and PHY-0722703), "Zwicky" at Caltech (funded by NSF MRI award PHY-0960291), "Finis Terrae" (funded by CESGA-ICTS-2010-200), "Caesaraugusta" (funded by BSC Grant Nos. AECT-2011-2-0006, AECT-2011-30007), "MareNostrum" (funded by BSC Grant Nos. AECT-2009-2-0017, AECT-20101-0008, AECT-2010-2-0013, AECT-2010-3-0010, AECT-2011-1-0015, AECT-2011-20012), "VSC" in Vienna (funded by FWF grant P22498), "Force" at GaTech, and on the GPC supercomputer at the SciNet HPC Consortium; SciNet is funded by: the Canada Foundation for Innovation under the auspices of Compute Canada; the Government of Ontario; Ontario Research Fund - Research Excellence; and the University of Toronto.

\section{Appendix A. Post-Newtonian Waveforms}

The accuracy of hybrid waveforms depends very sensitively on the accuracy of the post-Newtonian waveforms with which they are constructed. For this reason, the NINJA-2 collaboration invested significant effort into ensuring that all contributions used the most current $\mathrm{pN}$ information available. Mathematica notebooks containing the full expressions and derivations of the various approximants are provided in the ancillary files available with this paper online. Here, we describe the techniques.

The $\mathrm{pN}$ approximants used in this paper solve for the orbital motion to high accuracy by assuming that the motion is an adiabatic quasicircular inspiral, and by assuming that the loss of orbital binding energy $E$ during inspiral is balanced by the flux of energy in gravitational radiation to infinity $\mathcal{F}$ and the flux of energy going into the individual black holes caused by tidal heating $\dot{M}$. The first assumption means that the motion can be described completely by the orbital phase function $\Phi(t)$. We then define the pN expansion parameter $v:=(M \mathrm{~d} \Phi / \mathrm{d} t)^{1 / 3}$, where $M$ is the sum of the apparent-horizon masses of the black holes. The orbital binding energy, gravitationalwave flux, and tidal heating can be expressed as functions of this parameter. Thus, the energy-balance equation can be written as $\dot{E}+\mathcal{F}+\dot{M}=0$. Using the chain rule to rewrite $\dot{E}$, we can rearrange this as an expression for $\mathrm{d} v / \mathrm{d} t$, and include the expression for $\mathrm{d} \Phi / \mathrm{d} t$ to obtain a complete system of ordinary differential equations describing the motion of the binary:

$$
\frac{\mathrm{d} v}{\mathrm{~d} t}=-\frac{\mathcal{F}(v)+\dot{M}(v)}{E^{\prime}(v)} \quad \frac{\mathrm{d} \Phi}{\mathrm{d} t}=\frac{v^{3}}{M} .
$$

The formulation of the $\mathrm{pN}$ approximants, then, comes down to writing down the expressions for $E(v), \mathcal{F}(v)$, and $\dot{M}(v)$, and integrating the system for $v(t)$ and $\Phi(t)$.

At leading order, the expressions for $E$ and $\mathcal{F}$ are

$$
E(v)=-\frac{M \eta v^{2}}{2} \quad \text { and } \quad \mathcal{F}(v)=\frac{32}{5} v^{10} \eta^{2},
$$

where higher-order terms include additional factors of $v$. The additional terms are currently known up to $v^{7}(3.5 \mathrm{pN})$ for nonspinning systems, and to lower order for spinning systems. The tidal heating $\dot{M}$ is equivalent to a $2.5 \mathrm{pN}$ term in the flux 
expression. The full expressions for $E, \mathcal{F}$, and $\dot{M}$ are calculated in references [136142], with a collection of errata in reference [60], and are given explicitly in the accompanying Mathematica notebook PNOrbitalPhase.nb, which can be found in the ancillary material with this paper online. For all the results in this paper, the full expressions were used.

To integrate the system of ordinary differential equations, various methods have been developed, each of which should be equivalent at the level of accuracy of the $\mathrm{pN}$ approximation. These "approximants" have been given the names TaylorT1 through TaylorT4 $[29,143,144]$. The TaylorT1 approximant is constructed by directly evaluating the expressions in Eq. (A.1), which are then integrated numerically. For the TaylorT4 approximant, the right-hand side of the expression for $\mathrm{d} v / \mathrm{d} t$ is first re-expanded in a Taylor series, and truncated at the appropriate $3.5 \mathrm{pN}$ order, which is then integrated numerically. The difference between these approximants is at the level of the $4 \mathrm{pN}$ uncertainty.

Alternatively, we can find analytical formulas, rather than integrating numerically. The TaylorT2 approximant is derived by taking the (multiplicative) inverse of the first expression in Eq. (A.1) to construct a new system:

$$
\frac{\mathrm{d} t}{\mathrm{~d} v}=-\frac{E^{\prime}(v)}{\mathcal{F}(v)+\dot{M}(v)} \quad \frac{\mathrm{d} \Phi}{\mathrm{d} v}=\frac{\mathrm{d} \Phi}{\mathrm{d} t} \frac{\mathrm{d} t}{\mathrm{~d} v}=-\frac{v^{3}}{M} \frac{E^{\prime}(v)}{\mathcal{F}(v)+\dot{M}(v)}
$$

We re-expand the right-hand sides of these expressions in Taylor series, truncate at the appropriate $3.5 \mathrm{pN}$ order, and integrate with respect to $v$, obtaining expressions for $t(v)$ and $\Phi(v)$. This parametrically determines the phase of the binary as a function of time, with $v$ being the independent parameter. Finally, the TaylorT3 approximant is constructed by inverting the series $t(v)$ to obtain $v(t)$. There is a $3 \mathrm{pN}$ logarithmic term in $t(v)$, which must be treated as a constant in order to invert the series. Once this is done, the series for $v(t)$ can be inserted into $\mathrm{d} \Phi / \mathrm{d} t=v^{3} / M$, which can be integrated analytically to find $\Phi(t)$.

Using any of these approximants, the resulting orbital phase and frequency allow us to calculate the metric perturbation function $h$. The perturbation falls off at leading order as $1 / r$ and varies with angle, typically being decomposed in spin-weight $s=-2$ spherical harmonics [60]. The dominant mode of this decomposition is generally the $(\ell, m)=(2,2)$ mode. At leading order we have $\ddagger$

$$
h_{2,2}(v, \Phi)=\frac{M}{r} \sqrt{\frac{\pi}{5}} 8 \eta v^{2} \mathrm{e}^{-\mathrm{i} 2 \Phi},
$$

where higher-order terms include additional factors of $v$. The additional terms are currently known up to $v^{6}(3 \mathrm{pN})$ for nonspinning systems [145], and to lower order for spinning systems $[60,146]$. The full expressions used for this paper, including other modes, are given in the accompanying Mathematica notebook PNWaveform.nb found in the ancillary materials with this paper online.

$\ddagger$ Note that most references discuss a change of variables given by $\Psi:=\Phi-6 v^{3} \ln \left(v / v_{0}\right)$, which is a relative $4 \mathrm{pN}$ modification to the phase (and can therefore be ignored at the level of accuracy currently known for the phase evolution). Here, $v_{0}$ is a freely specifiable parameter related to the origin of the time coordinate. This modification removes related terms in expressions for the waveform amplitude by shifting them to terms at $4.5 \mathrm{pN}$ order and higher, which are currently unknown. We emphasize that - at the level of current $\mathrm{pN}$ knowledge - this new variable $\Psi$ never needs to be calculated explicitly from the known orbital phase and frequency. Where the expressions for $h$ refer to $\Psi$, the standard orbital phase $\Phi$ derived from the TaylorT $n$ approximants may be used directly in place of $\Psi$ without subtracting the logarithm, and any term involving $\ln \left(v / v_{0}\right)$ should be removed from the expressions for amplitude. 
The approximants just discussed describe the $\mathrm{pN}$ waveform in the time domain. For many purposes, it can be useful to have expressions in the frequency domain. The frequency-domain approximant TaylorF2 is obtained from TaylorT2 using the stationary-phase approximation (SPA) [147], together with the approximation that the gravitational-wave frequency is just twice the orbital frequency, so $f=v^{3} / \pi M$. Then the frequency-domain waveform is given by

$\tilde{h}_{\ell, m}(f)=h_{\ell, m}(v, 0) \sqrt{\frac{2 \pi M}{3 m v^{2} \dot{v}}} \exp \left\{\mathrm{i}\left[2 v^{3} t(v) / M-m \Phi(v)-\pi / 4\right]\right\}$,

where $\dot{v}$ is given in equation (A.1), and $t(v)$ and $\Phi(v)$ are the results of the TaylorT2 approximant.

\section{References}

[1] Abbott B et al. (LIGO Scientific) 2009 Rept. Prog. Phys. 72076901 (Preprint 0711.3041 )

[2] Shoemaker D (the Advanced LIGO Team) 2009 Advanced LIGO Reference Design [LIGOM060056]

[3] Harry G M and the LIGO Scientific Collaboration 2010 Class. Quant. Grav. 27084006

[4] Accadia T, Acernese F, Antonucci F, Astone P, Ballardin G et al. 2011 Class. Quant. Grav. 28114002

[5] The Virgo Collaboration 2009 Advanced Virgo Baseline Design [VIR-0027A-09]

[6] Kuroda K and the LCGT Collaboration 2010 Class. Quantum Grav. 27084004

[7] Thorne K S 1987 Three hundred years of gravitation ed Hawking S W and Israel W (Cambridge: Cambridge University Press) chap 9, pp 330-458

[8] Sathyaprakash B S and Schutz B F 2009 Living Rev. Relativity 12 (Preprint 0903.0338)

[9] Centrella J, Baker J G, Kelly B J and van Meter J R 2010 Rev. Mod. Phys. 823069 (Preprint 1010.5260)

[10] Aylott B, Baker J G, Boggs W D, Boyle M, Brady P R et al. 2009 Class. Quant. Grav. 26 165008 (Preprint 0901.4399 )

[11] Farr W M, Sravan N, Cantrell A, Kreidberg L, Bailyn C D et al. 2011 Astrophys. J. 741103 (Preprint 1011.1459)

[12] Özel F, Psaltis D, Narayan R and McClintock J E 2010 Astrophys. J. 725 1918-1927 (Preprint 1006.2834)

[13] Shoemaker, David and Losurdo, Giovanni 2010 Possible scenarios for commissioning and early observing with the second generation detectors Tech. Rep. LIGO-G10001760-v7 LIGO Project URL https://dcc.ligo.org/DocDB/0009/G1000176/007/Advanced\%20Virgo\%20and\% 20LIGO\%20post-project\%20progression.pdf

[14] The LIGO Scientific Collaboration 2009 Advanced ligo anticipated sensitivity curves Tech. Rep. LIGO-T0900288-v3 LIGO Project URL https://dcc.ligo.org/DocDB/0002/T0900288/003/ AdvLIG0\%20noise\%20curves.pdf

[15] URL http://www.ninja-project.org/

[16] Hannam M, Husa S, Ohme F and Ajith P 2010 Phys. Rev. D82 124052 (Preprint 1008.2961)

[17] Damour T, Nagar A and Trias M 2011 Phys. Rev. D83 024006 (Preprint 1009.5998)

[18] MacDonald I, Nissanke S, Pfeiffer H P and Pfeiffer H P 2011 Class. Quant. Grav. 28134002 (Preprint 1102.5128)

[19] Boyle M 2011 Phys. Rev. D84 064013 (Preprint 1103.5088)

[20] Ohme F, Hannam M and Husa S 2011 Phys. Rev. D84 064029 (Preprint 1107.0996)

[21] Hannam M et al. 2009 Phys. Rev. D79 084025 (Preprint 0901.2437)

[22] Lovelace G, Boyle M, Scheel M A and Szilagyi B 2011 Submitted to Class. Quantum Grav. (Preprint 1110.2229)

[23] Pfeiffer H P, Kidder L E, Scheel M A and Teukolsky S A 2003 Comput. Phys. Commun. 152 253-273 (Preprint gr-qc/0202096)

[24] Scheel M A et al. 2006 Phys. Rev. D74 104006 (Preprint gr-qc/0607056)

[25] Szilagyi B, Lindblom L and Scheel M A 2009 Phys. Rev. D80 124010 (Preprint 0909.3557)

[26] Lovelace G, Scheel M A and Szilagyi B 2011 Phys. Rev. D83 024010 (Preprint 1010.2777)

[27] Scheel M A, Boyle M, Chu T, Kidder L E, Matthews K D and Pfeiffer H P 2009 Phys. Rev. D79 024003

[28] Spectral Einstein Code URL http://www.black-holes.org/SpEC.html 
[29] Boyle M, Brown D A, Kidder L E, Mroue A H, Pfeiffer H P et al. 2007 Phys. Rev. D76 124038 (Preprint 0710.0158)

[30] Lindblom L, Scheel M A, Kidder L E, Owen R and Rinne O 2006 Class. Quant. Grav. 23 S447-S462 (Preprint gr-qc/0512093)

[31] Boyle M and Mroue A H 2009 Phys. Rev. D80 124045 (Preprint 0905.3177)

[32] Husa S, Gonzalez J A, Hannam M, Brügmann B and Sperhake U 2008 Class. Quant. Grav. 25 105006 (Preprint 0706.0740)

[33] Hannam M, Husa S, Bruegmann B and Gopakumar A 2008 Phys. Rev. D78 104007 (Preprint 0712.3787)

[34] Ajith P, Hannam M D, Husa S, Chen Y, Brügmann B, Dorband E N, Müller D, Ohme F, Pollney D, Reisswig C, Santamaría L and Seiler J 2009 "Complete" gravitational-waveform templates for black-hole binaries with non-precessing spins (Preprint 0909.2867)

[35] Hannam M, Husa S, Ohme F, Muller D and Bruegmann B 2010 Phys. Rev. D82 124008 (Preprint 1007.4789)

[36] Brügmann B et al. 2008 Phys. Rev. D77 024027 (Preprint gr-qc/0610128)

[37] Hannam M, Husa S, Sperhake U, Brugmann B and Gonzalez J A 2008 Phys. Rev. D77 044020 (Preprint 0706.1305)

[38] Chu T, Pfeiffer H P and Scheel M A 2009 Phys. Rev. D80 124051 (Preprint 0909.1313)

[39] Pollney D and Reisswig C 2011 Astrophys. J. 732 L13 (Preprint 1004.4209)

[40] Reisswig C, Bishop N T, Pollney D and Szilagyi B 2010 Class. Quant. Grav. 27075014 (Preprint 0912.1285)

[41] Pollney D, Reisswig C, Schnetter E, Dorband N and Diener P 2011 Phys. Rev. D83 044045 (Preprint 0910.3803)

[42] Healy J, Herrmann F, Hinder I, Shoemaker D M, Laguna P and Matzner R A 2009 Phys. Rev. Lett. 102041101 (Preprint 0807.3292 )

[43] Healy J, Laguna P, Matzner R A and Shoemaker D M 2010 Phys. Rev. D81 081501 (Preprint 0905.3914)

[44] Bode T, Haas R, Bogdanovic T, Laguna P and Shoemaker D 2010 Astrophys. J. 715 1117-1131 (Preprint 0912.0087)

[45] Herrmann F, Hinder I, Shoemaker D M, Laguna P and Matzner R A 2007 Phys. Rev. D76 084032 (Preprint 0706.2541)

[46] Healy J, Levin J and Shoemaker D 2009 Phys. Rev. Lett. 103131101 (Preprint 0907.0671)

[47] Bode T, Bogdanovic T, Haas R, Healy J, Laguna P et al. 2012 Astrophys. J. 74445 (Preprint $1101.4684)$

[48] Hinder I, Vaishnav B, Herrmann F, Shoemaker D and Laguna P 2008 Phys. Rev. D77 081502 (Preprint 0710.5167)

[49] Etienne Z B, Liu Y T, Shapiro S L and Baumgarte T W 2009 Phys. Rev. D79 044024 (Preprint 0812.2245)

[50] Buchman L T, Pfeiffer H P, Scheel M A and Szilagyi B Simulations of non-equal mass black hole binaries in preparation

[51] Tichy W and Marronetti P 2011 Phys. Rev. D83 024012 (Preprint 1010.2936)

[52] Brügmann B, González J, Hannam M, Husa S, Sperhake U and Tichy W 2008 Phys. Rev. D77 024027 (Preprint gr-qc/0610128)

[53] Marronetti P et al. 2007 Class. Quant. Grav. 24 S43-S58 (Preprint gr-qc/0701123)

[54] Brügmann B, Tichy W and Jansen N 2004 Phys. Rev. Lett. 92211101 (Preprint gr-qc/ 0312112)

[55] Sperhake U 2007 Phys. Rev. D76 104015 (Preprint gr-qc/0606079)

[56] Sperhake U et al. 2008 Phys. Rev. D78 064069 (Preprint 0710.3823)

[57] Lousto C O, Nakano H, Zlochower Y and Campanelli M 2010 Phys. Rev. Lett. 104211101 (Preprint 1001.2316)

[58] Campanelli M, Lousto C O, Marronetti P and Zlochower Y 2006 Phys. Rev. Lett. 96111101 (Preprint gr-qc/0511048)

[59] Lousto C O, Nakano H, Zlochower Y and Campanelli M 2010 Phys. Rev. D82 104057 (Preprint 1008.4360)

[60] Brown D A et al. 2007 Data formats for numerical relativity waves (Preprint 0709.0093)

[61] Tichy W and Marronetti P 2008 Phys. Rev. D78 081501 (Preprint 0807.2985)

[62] Zlochower Y, Baker J G, Campanelli M and Lousto C O 2005 Phys. Rev. D72 024021 (Preprint gr-qc/0505055)

[63] Lousto C O and Zlochower Y 2008 Phys. Rev. D77 024034 (Preprint 0711.1165)

[64] Vaishnav B, Hinder I, Herrmann F and Shoemaker D 2007 Phys. Rev. D76 084020 (Preprint $0705.3829)$ 
[65] Etienne Z B, Liu Y T, Shapiro S L and Baumgarte T W 2009 Phys. Rev. D79 044024 (Preprint 0812.2245)

[66] Farris B D, Liu Y T and Shapiro S L 2011 Phys. Rev. D84 024024 (Preprint 1105.2821)

[67] Etienne Z B, Liu Y T, Paschalidis V and Shapiro S L 2011 (Preprint 1112.0568)

[68] Baumgarte T W and Shapiro S L 2010 Numerical Relativity: Solving Einstein's Equations on the Computer (Cambridge University Press)

[69] Friedrich H and Rendall A D 2000 Lect. Notes Phys. 540 127-224 (Preprint gr-qc/0002074)

[70] Pretorius F 2005 Class. Quant. Grav. 22 425-452 (Preprint gr-qc/0407110)

[71] Cook G B and Pfeiffer H P 2004 Phys. Rev. D70 104016 (Preprint gr-qc/0407078)

[72] Caudill M, Cook G B, Grigsby J D and Pfeiffer H P 2006 Phys. Rev. D74 064011 (Preprint gr-qc/0605053)

[73] Lovelace G, Owen R, Pfeiffer H P and Chu T 2008 Phys. Rev. D78 084017 (Preprint 0805.4192)

[74] Nakamura T, Oohara K and Kojima Y 1987 Prog. Theor. Phys. Suppl. 90 1-218

[75] Shibata M and Nakamura T 1995 Phys. Rev D52 5428-5444

[76] Baumgarte T W and Shapiro S L 1999 Phys. Rev. D59 024007 (Preprint gr-qc/9810065)

[77] Baker J G, Centrella J, Choi D I, Koppitz M and van Meter J 2006 Phys. Rev. Lett. 96111102 (Preprint gr-qc/0511103)

[78] Bona C, Masso J, Seidel E and Stela J 1997 Phys. Rev. D56 3405-3415 (Preprint gr-qc/ 9709016)

[79] Hannam M, Husa S, Pollney D, Brügmann B and Ó Murchadha N 2007 Phys. Rev. Lett. 99 241102 (Preprint gr-qc/0606099)

[80] Hannam M et al. 2007 J. Phys. Conf. Ser. 66012047 (Preprint gr-qc/0612097)

[81] Brown J D 2008 Phys. Rev. D77 044018 (Preprint 0705.1359)

[82] Hannam M, Husa S, Ohme F, Brügmann B and Ó Murchadha N 2008 Phys. Rev. D78 064020 (Preprint 0804.0628)

[83] Alcubierre M et al. 2003 Phys. Rev. D67 084023 (Preprint gr-qc/0206072)

[84] van Meter J R, Baker J G, Koppitz M and Choi D I 2006 Phys. Rev. D73 124011 (Preprint gr-qc/0605030)

[85] Goodale T, Allen G, Lanfermann G, Massó J, Radke T, Seidel E and Shalf J 2003 Vector and Parallel Processing - VECPAR'2002, 5th International Conference, Lecture Notes in Computer Science (Berlin: Springer)

[86] Cactus computational toolkit URL http://www. cactuscode.org/

[87] Schnetter E, Hawley S H and Hawke I 2004 Class. Quant. Grav. 21 1465-1488 (Preprint gr-qc/0310042)

[88] Schnetter E Carpet: A mesh refinement driver for cactus URL http://www.carpetcode.org/

[89] Loffler F, Faber J, Bentivegna E, Bode T, Diener P et al. 2011 (Preprint 1111.3344)

[90] Einstein toolkit home page URL http://einsteintoolkit.org

[91] Thornburg J 2004 Class. Quant. Grav. 21 743-766 (Preprint gr-qc/0306056)

[92] Ansorg M, Brügmann B and Tichy W 2004 Phys. Rev. D70 064011 (Preprint gr-qc/0404056)

[93] Babiuc M, Szilagyi B, Hawke I and Zlochower Y 2005 Class. Quant. Grav. 22 5089-5108 (Preprint gr-qc/0501008)

[94] Babiuc M C, Bishop N T, Szilagyi B and Winicour J 2009 Phys. Rev. D79 084011 (Preprint 0808.0861)

[95] Matzner R A, Huq M F and Shoemaker D 1999 Phys.Rev. D59 024015 (Preprint gr-qc/ 9805023)

[96] Hinder I 2010 Class. Quant. Grav. 27114004 (Preprint 1001.5161)

[97] Pfeiffer H P Numerical simulations of compact object binaries in preparation

[98] Cook G B 2000 Living Rev. Rel. 35 (Preprint gr-qc/0007085)

[99] Bowen J M and York James W J 1980 Phys. Rev. D21 2047-2056

[100] Beig R and Murchadha N O 1994 Class. Quant. Grav. 11 419-430 (Preprint gr-qc/9304034)

[101] Brandt S and Brügmann B 1997 Phys. Rev. Lett. 78 3606-3609 (Preprint gr-qc/9703066)

[102] Dain S and Friedrich H 2001 Commun. Math. Phys. 222 569-609 (Preprint gr-qc/0102047)

[103] Pfeiffer H P et al. 2007 Class. Quant. Grav. 24 S59-S82 (Preprint gr-qc/0702106)

[104] York James W J 1999 Phys. Rev. Lett. 82 1350-1353 (Preprint gr-qc/9810051)

[105] Pfeiffer H P and York James W J 2003 Phys.Rev. D67 044022 (Preprint gr-qc/0207095)

[106] Mroue A H, Pfeiffer H P, Kidder L E and Teukolsky S A 2010 Phys. Rev. D82 124016 (Preprint 1004.4697)

[107] Blanchet L 2002 Living Rev. Rel. 53 (Preprint gr-qc/0202016)

[108] Schäfer G 2010 Post-Newtonian methods: Analytic results on the binary problem (New York: Springer) chap 6 (Preprint 0910.2857)

[109] Damour T and Nagar A 2010 The Effective One Body description of the Two-Body problem 
(New York: Springer) chap 7 (Preprint 0906.1769)

[110] Buonanno A, Kidder L E, Mroue A H, Pfeiffer H P and Taracchini A 2011 Phys. Rev. D83 104034 (Preprint 1012.1549)

[111] Pürrer M, Husa S and Hannam M 2011 A New Method to Reduce Eccentricity in NumericalRelativity Simulations of Black-hole-Binary Inspiral in preparation

[112] Boyle M and Mroué A H 2009 Phys. Rev. D80 124045

[113] Reisswig C, Bishop N, Pollney D and Szilagyi B 2009 Phys. Rev. Lett. 103221101 (Preprint $0907.2637)$

[114] Regge T and Wheeler J A 1957 Phys. Rev. 108 1063-1069

[115] Zerilli F J 1970 Phys. Rev. Lett. 24 737-738

[116] Sarbach O and Tiglio M 2001 Phys. Rev. D64 084016 (Preprint gr-qc/0104061)

[117] Rinne O, Buchman L T, Scheel M A and Pfeiffer H P 2009 Class. Quant. Grav. 26075009 (Preprint 0811.3593)

[118] Newman E and Penrose R 1962 J. Math. Phys. 3 566-578

[119] Reisswig C and Pollney D 2011 Class. Quant. Grav. 28195015 (Preprint 1006.1632)

[120] Damour T, Nagar A, Hannam M, Husa S and Brügmann B 2008 Phys. Rev. D78 044039 (Preprint 0803.3162)

[121] Baker J G, van Meter J R, McWilliams S T, Centrella J and Kelly B J 2007 Phys. Rev. Lett. 99181101 (Preprint gr-qc/0612024)

[122] Pretorius F 2005 Phys. Rev. Lett. 95121101 (Preprint gr-qc/0507014)

[123] Boyle M, Buonanno A, Kidder L E, Mroue A H, Pan Y et al. 2008 Phys. Rev. D78 104020 (Preprint 0804.4184)

[124] Santamaria L et al. 2010 Phys. Rev. D82 064016 (Preprint 1005.3306)

[125] Damour T, Nagar A, Dorband E N, Pollney D and Rezzolla L 2008 Phys. Rev. D77 084017 (Preprint 0712.3003)

[126] Pan Y, Buonanno A, Boyle M, Buchman L T, Kidder L E et al. 2011 (Preprint 1106.1021)

[127] Ajith P et al. 2008 Phys. Rev. D77 104017 (Preprint 0710.2335)

[128] Boyle M, Brown D A and Pekowsky L 2009 Class. Quant. Grav. 26114006 (Preprint $0901.1628)$

[129] Campanelli M et al. 2010 Class. Quant. Grav. 27084034 (Preprint 1001.3834)

[130] Sperhake U, Bruegmann B, Muller D and Sopuerta C 2011 Class. Quant. Grav. 28134004

[131] Ajith P et al. 2011 Phys. Rev. Lett. 106241101 (Preprint 0909.2867)

[132] Buonanno A, Iyer B, Ochsner E, Pan Y and Sathyaprakash B S 2009 Phys. Rev. D80 084043 (Preprint 0907.0700)

[133] Lindblom L, Owen B J and Brown D A 2008 Phys. Rev. D78 124020 (Preprint 0809.3844)

[134] Lindblom L, Baker J G and Owen B J 2010 Phys. Rev. D82 084020 (Preprint 1008.1803)

[135] Abadie J et al. (The LIGO Scientific Collaboration and the Virgo Collaboration, the Virgo Collaboration) 2011 Phys. Rev. D83 122005 (Preprint 1102.3781)

[136] Blanchet L, Faye G, Iyer B R and Joguet B 2002 Phys. Rev. D65 061501

[137] Blanchet L, Faye G, Iyer B R and Joguet B 2005 Phys. Rev. D71 129902

[138] Blanchet L, Iyer B R and Joguet B 2005 Phys. Rev. D71 129903

[139] Arun K G, Buonanno A, Faye G and Ochsner E 2009 Phys. Rev. D79 104023

[140] Blanchet L, Buonanno A and Faye G 2006 Phys. Rev. D74 104034 (Preprint gr-qc/0605140v4)

[141] Blanchet L, Buonanno A and Faye G 2007 Phys. Rev. D75 049903

[142] Blanchet L, Buonanno A and Faye G 2010 Phys. Rev. D81 089901

[143] Damour T, Iyer B R and Sathyaprakash B S 2001 Phys. Rev. D63 044023

[144] Buonanno A, Cook G B and Pretorius F 2007 Phys. Rev. D75 124018 (Preprint gr-qc/ 0610122)

[145] Blanchet L, Faye G, Iyer B R and Sinha S 2008 Class. Quantum Grav. 25165003

[146] Will C M and Wiseman A G 1996 Phys. Rev. D54 4813

[147] Cutler C and Flanagan É E 1994 Phys. Rev. D49 2658 